\title{
Multiple circulating alkaloids and saponins from intravenous Kang-Ai injection inhibit human cytochrome P450 and UDP-glucuronosyltransferase isozymes: potential drug-drug interactions
}

Zifei Qin $^{1,2,3}(0)$, Mengmeng Jia ${ }^{1,2}$, Jing Yang ${ }^{1,2^{*}}$, Han Xing ${ }^{1,2}$, Zhao Yin ${ }^{1,2^{*}}$, Zhihong Yao ${ }^{3,4}$, Xiaojian Zhang ${ }^{1,2}$ and Xinsheng $\mathrm{YaO}^{3,4}$

\begin{abstract}
Background: Kang-Ai injection is widely used as an adjuvant therapy drug for many cancers, leukopenia, and chronic hepatitis B. Circulating alkaloids and saponins are believed to be responsible for therapeutic effects. However, their pharmacokinetics (PK) and excretion in vivo and the risk of drug-drug interactions (DDI) through inhibiting human cytochrome P450 (CYP) and UDP-glucuronosyltransferase (UGT) enzymes remain unclear.

Methods: PK and excretion of circulating compounds were investigated in rats using a validated ultra-high-performance liquid chromatography tandem mass spectrometry (UHPLC-MS) method. Further, the inhibitory effects of nine major compounds against eleven CYP and UGT isozymes were assayed using well-accepted specific substrate for each enzyme.
\end{abstract}

Results: After dosing, 9 alkaloids were found with $C_{\max }$ and $t_{1 / 2}$ values of $0.17-422.70 \mu \mathrm{mol} / \mathrm{L}$ and $1.78-4.33 \mathrm{~h}$, respectively. Additionally, 28 saponins exhibited considerable systemic exposure with $t_{1 / 2}$ values of $0.63-7.22 \mathrm{~h}$, whereas other trace saponins could be negligible or undetected. Besides, over $90 \%$ of alkaloids were excreted through hepatobiliary and renal excretion. Likewise, astragalosides and protopanaxatriol (PPT) type ginsenosides also involved in hepatobiliary and/or renal excretion. Protopanaxadiol (PPD) type ginsenosides were mainly excreted to urine. Furthermore, PPD-type ginsenosides were extensively bound $\left(f_{u-p l a s m a}\right.$ approximately $1 \%$ ), whereas astragalosides and PPT-type ginsenosides displayed $f_{\text {u-plasma }}$ values of $12.35 \%$ and $60.23-87.36 \%$, respectively. Moreover, matrine, oxymatrine, astragaloside IV, ginsenoside Rg1, ginsenoside Re, ginsenoside Rd, ginsenoside Rc, and ginsenoside Rb1 exhibited no inhibition or weak inhibition against several common CYP and UGT enzymes $I_{50}$ values between 8.81 and $92.21 \mu \mathrm{M}$. Through kinetic modeling, their inhibition mechanisms towards those CYP and UGT isozymes were explored with obtained $\mathrm{K}_{\mathrm{i}}$ values. In vitro-in vivo extrapolation showed the inhibition of systemic clearance for CYP or UGT substrates seemed impossible due to [l]/K, no more than 0.1 .

Conclusions: We summarized the PK behaviors, excretion characteristics and protein binding rates of circulating alkaloids, astragalosides and ginsenosides after intravenous Kang-Ai injection. Furthermore, weak inhibition or no inhibition towards these CYP and UGT activities could not trigger harmful DDI when Kang-Ai injection is co-administered with clinical drugs primarily cleared by these CYP or UGT isozymes.

*Correspondence: jingyang_0101@163.com; yinzhao0601@163.com

1 Department of Pharmacy, The First Affiliated Hospital of Zhengzhou University, Zhengzhou 450052, China

Full list of author information is available at the end of the article

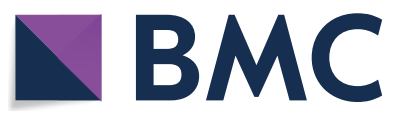

(c) The Author(s) 2020. This article is licensed under a Creative Commons Attribution 4.0 International License, which permits use, sharing, adaptation, distribution and reproduction in any medium or format, as long as you give appropriate credit to the original author(s) and the source, provide a link to the Creative Commons licence, and indicate if changes were made. The images or other third party material in this article are included in the article's Creative Commons licence, unless indicated otherwise in a credit line to the material. If material is not included in the article's Creative Commons licence and your intended use is not permitted by statutory regulation or exceeds the permitted use, you will need to obtain permission directly from the copyright holder. To view a copy of this licence, visit http://creativecommons.org/licenses/by/4.0/. The Creative Commons Public Domain Dedication waiver (http://creativecommons.org/publicdomain/zero/1.0/) applies to the data made available in this article, unless otherwise stated in a credit line to the data. 
Keywords: Kang-Ai injection, Pharmacokinetics, Excretion, Plasma protein binding rate, Cytochrome P450, UDPglucuronosyltransferase, Drug-drug interactions

\section{Background}

Herbal medicines are gaining popularity for the treatment of chronic and complex diseases as it works by hitting multiple molecular targets to modulate different signaling pathways [1-3]. Major advances have been made regarding their activities related their clinical indications, and more importantly in understanding their pharmacokinetics (PK) and excretion [3]. The PK profiles, just like the pharmacological activities, can be used as a "sieve" to assess the effectiveness of the individual compounds, and identify the chemical basis in an herbal injection [3-5]. Therefore, PK behaviors and excretion of circulating compounds should be first implemented in laboratory animals to reveal their systemic exposure. The corresponding PK parameters could show the ability of herbal compounds to be absorbed and transferred from the site of administration to the action targets through multiple biological barriers, and metabolic stability in vivo.

Kang-Ai injection is an herbal medicine approved in 2002 by the China Food and Drug Administration (China FDA). It consists of Astragali Radix (Huangqi), Ginseng Radix et Rhizoma (Renshen) and kushenin (Kushensu), and is indicated for many cancers, leukopenia and chronic hepatitis B [6-8]. Previously, we have finished the chemical identification and content determination of the main alkaloids (oxymatrine, matrine), astragalosides (astragaloside IV) and ginseng saponins (ginsenosides Rg1, Re) in Kang-Ai injection [9]. Despite the increasing understanding of Kang-Ai injection in chemical and pharmacological studies, the systemic exposure and excretion of its bioactive compounds after administration remain to be elucidated.

Previous PK researches of individual herb or its characteristic compounds have been carried out, which greatly facilitated the holistic PK properties of Kang-Ai injection. Oxymatrine, the most abundant herbal compound in Kang-Ai injection, occupies over $95.37 \%$ of total contents. When intravenously treated with oxymatrine at $30 \mathrm{mg} / \mathrm{kg}$, the $\mathrm{AUC}_{0-12 \mathrm{~h}}, C_{\max }, t_{1 / 2}$ and $\mathrm{CL}$ values in rat plasma were $16234.27 \mathrm{ng} \mathrm{h} / \mathrm{mL}, 32847.57 \mathrm{ng} / \mathrm{mL}, 0.57 \mathrm{~h}$ and $3.10 \mathrm{~L} / \mathrm{h}$, respectively [10]. Besides, only about $19.9 \%$ of oxymatrine was reduced to matrine following intravenous infusion of $600 \mathrm{mg}$ oxymatrine in healthy male volunteers [11]. In addition, systemic exposure of astragaloside IV appeared to be dose proportional over the dose ranged from 200 to $500 \mathrm{~mL}$ astragaloside IV $(9 \mathrm{mg} / 100 \mathrm{~mL})$ in healthy volunteers [12]. Furthermore, metabolism of astragaloside IV by intestinal microbiome was a crucial step in its excretion, rather than by hepatic and intestinal enzymes [13-15]. Moreover, ginsenosides $\mathrm{Rg} 1, \mathrm{Re}$, and Rf were not extensively bound in plasma, and also were with substantially shorter $t_{1 / 2}$ values and significantly larger systemic clearance $\left(\mathrm{CL}_{\text {tot, } \mathrm{p}}\right)$ than other-type ginsenosides [16]. Although several bioanalytical assays have been applied to the PK study of several circulating alkaloids and saponins, PK and excretion information about Kang-Ai injection is still limited. This also brings a huge obstacle in our understanding of the chemical basis responsible for the therapeutic effects of Kang-Ai injection.

In addition, drug-drug interaction (DDI) is significant safety concerns in clinical medication [17-19]. Inhibition or induction of human CYP or UGT isozymes could potentially adverse clinical DDI and result in metabolic disorders for endogenous components [20-22]. It would be more serious for the clinical drugs with narrow therapeutic indices, such as digoxin, warfarin, $\mathrm{SN}-38$ and so on [17]. Similarly, UGT1A1 is the only one UGT isozyme in the conjugative detoxification of bilirubin [21]. Once UGT1A1 function is inhibited, there is a high risk of liver injury [21]. Considering the intravenous administration of Kang-Ai injection and abundant exposure of its circulating herbal compounds, it should be of particularly clinical significance to investigate the potential risks of DDI.

To this goal, this current report first identified the major circulating alkaloids, astragalosides, ginsenosides in rat samples after an intravenous 30 min-infusion of Kang-Ai injection at $6 \mathrm{~mL} / \mathrm{kg}$. In addition, the detailed PK behaviors, and exposure of these herbal substances in plasma were systemically carried out. Meanwhile, we also investigated the plasma binding rate assays of these circulating alkaloids and saponins. Furthermore, the excretion of these compounds in urine and bile were determined by dividing the cumulative amount excreted into urine (Cum. $\left.A_{\mathrm{e}-\mathrm{U}}\right)$ and bile (Cum. $A_{\mathrm{e}-\mathrm{B}}$ ) by their respect doses. Moreover, we explored the inhibitory effects and mechanism of nine major circulation compounds against several important CYP and UGT enzymes based on their widely recognized probe substrates. Taken together, the current PK and excretion information would facilitate the identification of active herbal compounds responsible for the therapeutic actions of Kang-Ai injection, and would also be helpful to inform its potential DDI in rational clinical use and achieve the optimal herbal therapy. 


\section{Materials and methods Chemicals and reagents}

Kang-Ai injection samples (lot numbers of 03180805, 03181004, 01180908, 01180909, 02190102, 02190103) were manufactured by Changbaishan Pharmaceutical Co., Ltd. (Jilin, China) with China FDA drug ratification number of GuoYaoZhunZiZ-20026868. Purified matrine, oxymatrine, oxysophocarpine, isoastragaloside IV, and astragaloside III, IV, V, VI were all purchased Guangzhou Fans Biotechnology Co., Ltd (Guangzhou, China). Reference standards of ginsenosides Rb1, Rb2, Rc, Rd, Re, Rf, Rg1, Rg2, Rg6, Rh1, Rh4, Rk3, F1, F2, F3, F4, and notoginsenosides R1, R2 were all obtained from Shanghai Yuanye Biotechnology Co., Ltd. (Shanghai, China). The purity of these compounds was over $98.0 \%$.

Magnesium chloride $\left(\mathrm{MgCl}_{2}\right)$, D-saccharic-1, 4-lactone monohydrate, alamethicin, nicotinamide adenine dinucleotide phosphate (NADPH), uridine 5'-diphospho-glucuronosyltransferase (UDPGA) were all obtained from Sigma-Aldrich (St. Louis, MO, USA). 4-hydroxytolbutamide, 4-hydroxymephenytoin, 4-methylumbelliferone (4-MU), 6 $\alpha$-hydroxy-paclitaxel, 6-hydroxychlorzoxazone, 7-hydroxycoumarin, $\beta$-estradiol, bupropion, coumarin, chlorzoxazone, hydroxybupropion, mephenytoin, nifedipine, oxidized nifedipine, phenacetin, paracetamol, paclitaxel, propofol and tolbutamide were all acquired from Aladdin Chemicals (Shanghai, China). 4-MU-glucuronide, $\beta$-estradiol-3-O-glucuronide and propofol$\mathrm{O}$-glucuronide were all obtained from Toronto Research Chemicals (North York, ON, Canada). Recombinant CYP1A2 (Cat. No. 456203), CYP2A6 (Cat. No. 456254), CYP2B6 (Cat. No. 456255), CYP2C8 (Cat. No. 456252), CYP2C9 (Cat. No. 456258), CYP2C19 (Cat. No. 456259), CYP2E1 (Cat. No. 456206), CYP3A4 (Cat. No. 456202), and expressed UGT1A1 (Cat. No. 456411), UGT1A9 (Cat. No. 456419), UGT2B7 (Cat. No. 456427) were all provided from Corning Biosciences (Corning, NY, USA).

LC-MS-grade water, methanol and acetonitrile were purchased from Fisher Scientific (Fair Lawn, New Jersey, USA). LC-MS grade formic acid was obtained from Sigma-Aldrich (St. Louis, USA). Other reagents were of analytical or higher grade.

\section{Animals}

Specific pathogen free (SPF) grade male Sprague-Dawley rats $(250 \pm 20) \mathrm{g}$ were provided by the Experimental Animal Center of Zhengzhou University (Zhengzhou, China). These rats were kept in a designated animal room at constant temperature $(22 \pm 2){ }^{\circ} \mathrm{C}$ and humidity $(50 \pm 20) \%$ with $12 \mathrm{~h}$ of light/dark per day and free access to water and food. All protocols of animal experiments were approved in accordance with the Regulations of Experimental Animal Administration issued by the
Ethics Review Committee for Animal Experimentation of Jinan University (Ethical Review NO. 20190301010). Prior to the experiments, the rats were fasted for $12 \mathrm{~h}$ but with free access to water. After study use, the rats were euthanized with $\mathrm{CO}_{2}$ gas.

\section{Sample preparation}

Each plasma sample $(50 \mu \mathrm{L})$ was transferred to a $1.5-\mathrm{mL}$ polypropylene tube containing $50 \mu \mathrm{L}$ of internal standard (IS) solution (including $20 \mathrm{nM}$ olanzapine-d3 and $1000 \mathrm{nM}$ mycophenolic acid-d3) and $50 \mu \mathrm{L}$ of methanol. The mixture was vortex mixed for $30 \mathrm{~s}$, and then 150 $\mu \mathrm{L}$ methanol was precipitated. The tubes were vortex mixed vigorously for $1 \mathrm{~min}$ and centrifuged at $13,800 \mathrm{~g}$ for $10 \mathrm{~min}$ at $4{ }^{\circ} \mathrm{C}$. Finally, $4 \mu \mathrm{L}$ aliquots of the supernatant was injected into the UHPLC/TQD-MS system. Similarly, rat urine $(50 \mu \mathrm{L})$ and bile $(50 \mu \mathrm{L})$ samples were precipitated with methanol at a volumetric sample-tomethanol ratio of 1:3; after centrifugation, the resulting supernatants were applied for analysis. All the biological samples were stored at $-80^{\circ} \mathrm{C}$.

\section{Preparation of standard solutions and quality control samples}

An appropriate amount of each authentic standard was separately dissolved with $60 \%$ methanol-water in a $10 \mathrm{~mL}$ volumetric flask to prepare the standard solution. Furthermore, an appropriate volume of each standard solution was transferred into a $10 \mathrm{~mL}$ volumetric flask to the desired concentration as the stock solution. The mixed IS solution contained olanzapine- $\mathrm{d} 3$ and mycophenolic acid-d3 at final concentration of $20 \mathrm{nM}$ and $1000 \mathrm{nM}$, respectively. All the solutions were stored at $4{ }^{\circ} \mathrm{C}$.

Calibration standard samples were prepared by spiking $50 \mu \mathrm{L}$ blank plasma (or other biological samples) with 50 $\mu \mathrm{L}$ working solutions and $50 \mu \mathrm{L}$ IS solution. Therefore, the plasma concentration ranges were $10-1000 \mathrm{nM}$ for matrine (A1), 10-1000 nM for oxysophocarpine (A2), 40-4000 nM for oxymatrine (A3), 40-4000 nM for astragaloside IV (B2), 10-1000 nM for astragaloside III (B3), 10-1000 nM for ginsenoside Rh1 (C1), 10-1000 nM for notoginsenoside R2 (C3), 10-1000 nM for ginsenoside Rg2 (C5), 20-2000 nM for ginsenoside Rg1 (C6), 10-1000 $\mathrm{nM}$ for ginsenoside Rf (C7), 10-1000 nM for notoginsenoside R1 (C8), 20-2000 $\mathrm{nM}$ for ginsenoside Re (C9), 10-1000 nM for ginsenoside Rd (D2), 10-1000 $\mathrm{nM}$ for ginsenoside Rc (D3), $10-1000 \mathrm{nM}$ for ginsenoside $\mathrm{Rb} 2$ (D4), 10-1000 $\mathrm{nM}$ for ginsenoside $\mathrm{Rb} 1$ (D5), respectively. Quality control (QC) samples were independently prepared in the same way at $2,10,40$ times of LLOQ (lower limit of quantification) of each analyte. The calibration standard and QC samples were 
processed on each analysis day with the same procedures for plasma samples.

\section{Chromatographic and mass spectrometric conditions} Ultra-high-performance liquid chromatography (UHPLC) analysis was performed on a Waters Acquity UHPLC I-Class system (Manchester, UK) for the quantitative analysis of Kang-Ai injection. Chromatographic separation was achieved on an BEH C18 column $(2.1 \times 50 \mathrm{~mm}, 1.7 \mu \mathrm{m})$ with water (A) and acetonitrile (B) (both including $0.1 \%$ formic acid) at a flow rate of $0.45 \mathrm{~mL} / \mathrm{min}$ with column temperature of $35^{\circ} \mathrm{C}$. The gradient flow profile was optimized as follows: $10 \%$ B from 0 to $0.5 \mathrm{~min}, 10-20 \% \mathbf{B}$ from 0.5 to $1.5 \mathrm{~min}$, maintaining $20 \% \mathbf{B}$ from 1.5 to $4.5 \mathrm{~min}, 20-30 \%$ B from 4.5 to $5.5 \mathrm{~min}$, $30-35 \%$ B from 5.5 to $7.5 \mathrm{~min}, 35-90 \%$ B from 7.5 to 9.5 min, keeping $90 \%$ B from 9.5 to $10.5 \mathrm{~min}, 90-10 \%$ B from 10.5 to $11.0 \mathrm{~min}$, and maintaining $10 \% \mathbf{B}$ from 11 to $12 \mathrm{~min}$.

UHPLC system was coupled to a triple quadrupole mass spectrometer (Waters Xevo TQD, Waters, Manchester, UK). The detailed mass spectrometers were adjusted as follows: capillary voltage, $3.5 \mathrm{kV}(\mathrm{ESI}+)$ or $1.5 \mathrm{kV}$ (ESI-); cone voltage, $50 \mathrm{~V}$ (ESI+) or $50 \mathrm{~V}$ (ESI-); source temperature, $350{ }^{\circ} \mathrm{C}$; desolvation gas flow, 650 $\mathrm{L} / \mathrm{h}$; The mass spectrometer was performed in the multiple reaction monitoring mode (MRM) using both positive and negative ionization. The quantitative parameters in MRM modes were same as our previous study. All experimental data were collected and processed using a Quanlynx software in Masslynx 4.1 platform.

\section{Method validation}

The developed UHPLC-MS/MS method was validated according to the Guidance for Industry: bioanalytical method validation from the US FDA for specificity, linearity, LLOQ, extraction recovery, matrix effects, precision, accuracy, stability and dilution integrity [23]. The assay validation of each analyte in rat biological samples including plasma, urine and bile were all performed based on the standard specification above.

Specificity was determined by comparing the chromatograms of blank samples (from six individual rats), with dosed samples after an intravenous bolus of Kang-Ai injection, and blank samples spiked with each analyte at LLOQ and IS. The calibration curves were constructed by the peak area ratios of the analytes to IS (Y), versus respective sample concentrations $(\mathrm{X})$ applying a weighted $\left(1 / x^{2}\right)$ least squares linear regression analysis. LLOQ was determined based on the lowest concentrations for which acceptable linearity, accuracy and precision were demonstrated. The accuracies (the relative error, RE) and inter/ intra-day precisions (the relative standard deviation,
RSD) of the assay were evaluated by determining six replicates of QC samples (at low, middle, and high concentrations) on three consecutive days.

Extraction recoveries (ER) and matrix effects (ME) were evaluated by a published experimental protocol [24]. The response of extracted QC sample was defined as A1. In addition, A2 referred to the corresponding standard solutions added into the post-extracted supernatant from biological matrix, while A3 corresponded to the responses of each analyte at three QC levels. The ER and ME values were calculated as follows: $E R \%=A 1 /$ $\mathrm{A} 2 \times 100 \%$, and $\mathrm{ME} \%=\mathrm{A} 2 / \mathrm{A} 3 \times 100 \%$.

Stability tests were investigated at three QC levels under different conditions, including short-term stability ( $8 \mathrm{~h}$ exposure at room temperature), autosampler storage stability of the methanol-treated samples (at $8{ }^{\circ} \mathrm{C}$ for $18 \mathrm{~h}$ ), freeze/thaw stability (three $-80{ }^{\circ} \mathrm{C} \leftrightarrow 23{ }^{\circ} \mathrm{C}$ cycles), long-term stability (14 days storage at $-80^{\circ} \mathrm{C}$ ). The dilution integrity was investigated by analyzing six replicate samples with each analyte, and each analyte were diluted 10-fold with blank rat matrix. Furthermore, the diluted samples were analyzed using freshly prepared calibration curve to calculate RE and RSD, which should be no more than $\pm 15 \%$ as criterion.

\section{In vivo rat studies}

The rats in this study all received a single 30-min intravenous infusion of Kang-Ai injection via the tail veins using TYD01-02 infusion pumps (Lead Fluid, Baoding, China). The dose was derived from the label daily dose of Kang-Ai injection $(60 \mathrm{~mL} /$ person, once daily) according to dose normalization by body surface area [25]. For plasma pharmacokinetic studies, the rats were randomly received a single intravenous $30 \mathrm{~min}$-bolus dose at $6 \mathrm{~mL} /$ $\mathrm{kg}$. Blood samples $(100 \mu \mathrm{L})$ were collected from the rats' external jugular veins into heparinized polypropylene tubes at 5, 15, 30, $45 \mathrm{~min}$, and 1, 1.5, 2, 3, 4, 6, 8, $12 \mathrm{~h}$. After gently shaking for $10 \mathrm{~s}$, the blood samples were centrifuged at $13000 \mathrm{~g}$ for $10 \mathrm{~min}$ to yield plasma samples, which were kept frozen at $-80^{\circ} \mathrm{C}$ until analysis.

For urine sampling, the rats were housed singly in metabolic cages. And then, the urine samples were collected at $0-4,4-8$ and $8-12 \mathrm{~h}$ after starting infusion and were weighed. Likewise, bile samples were also collected at $0-3,3-6,6-9$ and $9-12 \mathrm{~h}$ after starting infusion and were weighed. The urine and bile collection tubes were all frozen at $-80^{\circ} \mathrm{C}$.

\section{Protein binding assay}

Binding of circulating alkaloids and saponins to rat plasma was measured by equilibrium dialysis using Spectra/Por 2 dialysis membranes (molecular weight cutoff, 12-14 kDa; Rancho Dominguez, CA, USA) as described 
[26]. After equilibrating for $24 \mathrm{~h}$ at $55 \mathrm{rpm}$ and $37^{\circ} \mathrm{C}$, the dialysate and the plasma were sampled for analysis. In brief, the concentrations of each analyte in dialysate and rat plasma samples were determined for analysis after equilibrating at $55 \mathrm{rpm}$ and $37^{\circ} \mathrm{C}$ for $24 \mathrm{~h}$. The concentrations of tested matrine, oxysophocarpine and oxymatrine were $2 \mu \mathrm{mol} / \mathrm{L}$, while those of astragaloside IV, III, and ginsenosides Rh1, Rg2, Rg1, Rf, Re, Rd, Rc, Rb1, Rb2, notoginsenosides R1, R2, were $4 \mu \mathrm{mol} / \mathrm{L}$. The unbound fraction $\left(f_{\text {u-plasma }}\right)$ in rat plasma was calculated as follow: $f_{\text {u-plasma }}=C_{\mathrm{d}} / C_{\mathrm{p}} \times 100 \%$, where the $C_{\mathrm{d}}$ and $C_{\mathrm{p}}$ values were the concentrations of each analyte in the dialysate sample and in the post-dialysis plasma sample, respectively.

\section{Incubation systems}

Phase I incubation system $(100 \mu \mathrm{L})$ contained Tris- $\mathrm{HCl}$ buffer $(50 \mathrm{mM}, \mathrm{pH}=7.4), \mathrm{MgCl}_{2}(5 \mathrm{mM})$, each CYP isozyme, specific substrates, circulating compounds and NADPH $(1 \mathrm{mM})$ as prescribed previously [18]. After incubation $\left(37^{\circ} \mathrm{C}, 60 \mathrm{~min}\right)$, the reaction was terminated by ice-cold acetonitrile $(100 \mu \mathrm{L})$, following by centrifugation at $13,800 \mathrm{~g}$ for $10 \mathrm{~min}$. The supernatant $(8 \mu \mathrm{L})$ was acquired for ultra-high-performance liquid chromatography (UHPLC) system (Waters, Manchester, UK) analysis.

For glucuronidation assays, the incubation system (100 $\mu \mathrm{L})$ contained Tris $-\mathrm{HCl}$ buffer $(50 \mathrm{mM}, \mathrm{pH}=7.4)$, alamethicin $(22 \mu \mathrm{g} / \mathrm{mL}), \mathrm{D}$-saccharic-1, 4-lactone $(4.4 \mathrm{mM})$, each UGT enzyme, specific substrates, circulating compounds and UDPGA $(3.5 \mathrm{mM})$ as described recently [18]. The reaction was terminated by ice-cold acetonitrile $(100 \mu \mathrm{L})$ after incubation at $37^{\circ} \mathrm{C}$ for $60 \mathrm{~min}$. After centrifugation $(13,800 g, 10 \mathrm{~min})$, the supernatant $(8 \mu \mathrm{L})$ was obtained for determination by UHPLC system.

\section{Analysis of inhibitory effects}

In this study, phenacetin $(100 \mu \mathrm{M})$, coumarin $(100 \mu \mathrm{M})$, bupropion $(100 \mu \mathrm{M})$, paclitaxel $(60 \mu \mathrm{M})$, tolbutamide $(200 \mu \mathrm{M})$, mephenytoin $(100 \mu \mathrm{M})$, chlorzoxazone $(200 \mu \mathrm{M})$ and nifedipine $(40 \mu \mathrm{M})$ have been used as the probe substrates for CYP1A2, 2A6, 2B6, 2C8, 2C9, 2C19, 2E1 and 3A4, respectively [27]. After optimization of incubation conditions (Additional file 1: Fig. S1), the substrates were incubated with each CYP isozyme at different protein concentrations $(0.05 \mathrm{mg} / \mathrm{mL}$ for CYP1A2 and 3A4; $0.1 \mathrm{mg} / \mathrm{mL}$ for CYP2A6, 2B6, 2C8, 2C9, 2C19 and $2 \mathrm{E} 1$ ) in the absence (control) and presence of different mixture or herbal compounds (Kang-Ai injection and samples that Kang-Ai injection was diluted 10 times; 1 , 10 , and $100 \mu \mathrm{M}$ for matrine and oxymatrine; $0.1,1,10$ and $100 \mu \mathrm{M}$ for astragalosides and ginsenosides).

Similarly, $\beta$-estradiol $(60 \mu \mathrm{M})$, propofol $(40 \mu \mathrm{M})$ and 4-MU $(350 \mu \mathrm{M})$ were typically used as the substrates for UGT1A1, 1A9 and 2B7, respectively [28]. After optimization of incubation conditions (Additional file 1: Fig. S2), the protein concentrations for UGT1A1, 1A9, and $2 \mathrm{~B} 7$ were $0.125,0.05$, and $0.05 \mathrm{mg} / \mathrm{mL}$, respectively. Kang-Ai injection and samples that Kang-Ai injection was diluted 10 times, were both used to investigate the inhibitory effects against UGT1A1, 1A9 and 2B7. In addition, the concentrations were 1,10 and $100 \mu \mathrm{M}$ for matrine and oxymatrine, and $0.1,1,10$ and $100 \mu \mathrm{M}$ for astragalosides and ginsenosides based on the detailed plasma concentration after intravenous Kang-Ai injection.

The half-inhibition concentration $\left(\mathrm{IC}_{50}\right)$ values were determined by non-linear regression analysis. The inhibitory effects towards each CYP or UGT enzyme were divided into four categories as follows, potent $\left(\mathrm{IC}_{50}<1 \mu \mathrm{M}\right)$, moderate $\left(1 \mu \mathrm{M}<\mathrm{IC}_{50}<10 \mu \mathrm{M}\right)$, weak $\left(10 \mu \mathrm{M}<\mathrm{IC}_{50}<100 \mu \mathrm{M}\right)$, or no inhibition $\left(\mathrm{IC}_{50}>100 \mu \mathrm{M}\right)$ [18]. The inhibition mechanism towards corresponding CYP and UGT isoforms were further explored.

\section{Inhibition kinetic analysis}

The inhibition constant $\left(\mathrm{K}_{\mathrm{i}}\right)$ values were obtained by multiple concentrations of substrates in the absence or presence of multiple concentrations of herbal compounds as described previously $[18,27,28]$. Competitive inhibition, noncompetitive inhibition, and mixed-type inhibition models were used to determine the $\mathrm{K}_{\mathrm{i}}$ values by nonlinear regression analysis using the Eqs. (1)-(3), respectively. The model with the smallest Akaike information criterion (AIC) and Schwartz information criterion (SC) values was considered as the best model, following the obtained appropriate $\mathrm{K}_{\mathrm{i}}$ values. Model fitting and parameter estimation were performed using Graphpad Prism V5 software (SanDiego, CA).

The detailed parameters for three equations were as follow. $V$ is the velocity of the reaction. The $[\mathrm{S}]$ and [I] are the concentrations of substrate and herbal compounds, respectively. $K_{i}$ is the constant describing the affinity between herbal compounds and the enzyme. $K_{m}$ is the substrate concentration at half of the maximum velocity $\left(\mathrm{V}_{\max }\right)$ of the reaction. The $\alpha \mathrm{Ki}$ describes the affinity of herbal compounds to the complex of enzyme and substrate; When $\alpha$ is very large $(\alpha \gg 1)$, the binding of inhibitor would prevent the binding of substrate, and the mixed inhibition model becomes identical to competitive inhibition.

$$
\begin{aligned}
& V=\frac{V_{\max } \times[S]}{K_{m} \times\left(1+\frac{[I]}{K_{i}}\right)+[S]} \\
& V=\frac{V_{\max } \times[S]}{\left(K_{m}+[S]\right) \times\left(1+\frac{[I]}{K_{i}}\right.}
\end{aligned}
$$




$$
V=\frac{V_{\max } \times[S]}{K_{m} \times\left(1+\frac{[I]}{K_{i}}\right)+[S] \times\left(1+\frac{[I]}{\alpha K_{i}}\right)}
$$

\section{Data processing}

Pharmacokinetic parameters were estimated by noncompartmental analysis using WinNonlin 6.3 software (Pharsight, NC, US). Data are presented as the mean $\pm S D(n=3)$. The area under the concentrationtime curve up to the last measured point in time (AUC ${ }_{0-t}$ ) was calculated using the trapezoidal rule. The total plasma clearance $\left(\mathrm{CL}_{\text {tot, } \mathrm{p}}\right)$ was calculated by dividing the compound dose by the $\mathrm{AUC}_{0-\infty}$, and the distribution volume at steady state $\left(V_{\mathrm{SS}}\right)$ was estimated by multiplying the $\mathrm{CL}_{\text {tot, } \mathrm{p}}$ by the mean residence time (MRT). The renal excretory clearance $\left(\mathrm{CL}_{R}\right)$ and hepatobiliary $\left(\mathrm{CL}_{\mathrm{B}}\right)$ was determined by dividing the cumulative amount excreted into urine (Cum. $\left.A_{\mathrm{e}-\mathrm{U}}\right)$ and bile $\left(\right.$ Cum. $\left.A_{\mathrm{e}-\mathrm{B}}\right)$ by the plasma $\mathrm{AUC}_{0-\infty}$, respectively. The fractions of dose excreted into urine $\left(f_{\mathrm{e}-\mathrm{U}}\right)$ and the fractions of dose excreted into bile $\left(f_{\mathrm{e}-\mathrm{B}}\right)$ were established using the relationship Cum. $A_{\mathrm{e}-\mathrm{U}} /$ Dose and Cum. $A_{\mathrm{e}-\mathrm{B}} /$ Dose, respectively. Mean differences between treatment and control groups were analyzed by two-tailed unpaired Student's t test by Graphpad Prism V5 software (SanDiego, CA). The level of significance was set at $\mathrm{p}<0.05(*), p<0.01(* *)$ or $p<0.001(* * *)$.

\section{Results}

\section{Xenobiotics detected in rat samples}

After dosing Kang-Ai injection to rats $(6 \mathrm{~mL} / \mathrm{kg})$, a total of 9 circulating alkaloids, 6 astragalosides and 22 ginsenosides were detected and characterized in rat samples (Additional file 1: Fig. S3A and Table S1). Based on their individual content level from Kang-Ai injection [9], these compounds were graded into five dose levels (Additional file 1: Fig. S3B and Table S1): Level I was over $1000 \mu \mathrm{g} /$ $\mathrm{mL}$ for only oxymatrine (A3); Level II was between 100 and $1000 \mu \mathrm{g} / \mathrm{mL}$ for ginsenosides Rg1 (C6) and Re (C9); Level III was ranged from 10 to $100 \mu \mathrm{g} / \mathrm{mL}$ for matrine (A1), astragaloside IV (B2), notoginsenoside R2 (C3), ginsenoside $\operatorname{Rg} 2$ (C5), Rf (C7) and Rc (D3); Level IV was from 1 to $10 \mu \mathrm{g} / \mathrm{mL}$ for astragaloside III (B3), VI (B4), V (B5), notoginsenoside R1 (C8), ginsenoside Rh1 (C1), Rd (D2), Rb2 (D4), Rb1 (D5), Rh4 (E2), Rg6 (E3), F4 (E4); Level $\mathrm{V}$ was all less than $1 \mu \mathrm{g} / \mathrm{mL}$ for oxysophocarpine (A2), isoastragaloside IV (B1), ginsenoside F1 (C2), F3 (C4), F2 (D1), Rk3 (E1).

\section{Method validation}

The typical MRM chromatograms obtained from blank plasma, blank plasma spiked with the analyte (LLOQ), and dosed rat plasma after intravenous Kang-Ai injection
(30 min) were presented in Additional file 1: Fig. S4. As seen from MRM chromatograms, there were no endogenous interference for each analyte and two IS compounds, which suggested that the specificity of the quantitative method was satisfactory.

Additional file 1: Table S2 summarized the mean values of linear regression equation of the sixteen compounds. The correlation coefficients $\left(r^{2}\right)$ were ranged from 0.9900 to 0.9994 , which exhibited good linearity. The LLOQs of each circulation compound were between 5 and $40 \mathrm{nM}$, with the accuracies and precisions less than $20 \%$, which were enough for the pharmacokinetics study of sixteen analytes following a 30-min infusion of Kang-Ai injection to rats.

The results of the intra- and inter-day precision and accuracy of all analytes and three QC samples were displayed in Additional file 1: Table S3. The intraday and inter-day precision (RSD) of sixteen analytes were within the range from 2.35 to $12.64 \%$, whereas the accuracy (RE) derived from three QC samples was between -14.30 and $13.66 \%$, all meeting the requirements. These results proved that the developed method was accurate and reproducible.

Additional file 1: Table S4 exhibited the matrix effect and extraction recovery results of all analytes and two IS. The mean matrix effects of 16 circulation compounds were in the range from 92.06 to $110.45 \%$ with RSD values less than $14.14 \%$, and the average absolute matrix effects of olanzapine-d3 (IS-1) and mycophenolic acid-d3 (IS-2) were $100.29 \%$ and $109.12 \%$ with RSD values of $11.42 \%$ and $3.13 \%$, respectively. Similarly, the mean extraction recoveries ranged from $82.85 \%$ to $108.98 \%$ with RSD values no more than $14.73 \%$ at three QC levels, whereas the mean absolute recoveries of IS- 1 and IS- 2 were $80.54 \%$ and $83.60 \%$ with RSD values of $2.11 \%$ and $5.19 \%$, respectively. These results demonstrated that the recoveries obtained were consistent and reproducible, and the ionization competition (enhancement or suppression effects) between the analytes and the endogenous co-elution was negligible.

The mean stability results (Additional file 1: Table S5) indicated the RE and RSD values of sixteen circulating alkaloids and saponins at three QC levels were in the range from $-14.53 \%$ to $14.40 \%$ and $13.87 \%$, respectively, indicating that all the analytes were stable under different storage conditions.

In addition, a total of twenty circulating compounds were detected in rat urine. The regression equations, linear ranges and LLOQs for the determination of the analytes in rat urine was shown in Additional file 1: Table S6. Over the considered calibration range, the regression coefficient $\left(r^{2}\right)$ was $>0.9906$ for the curves of each analyte. Furthermore, twenty-one circulating 
compounds were obtained in rat bile, and the calibration curves of twenty-one analytes exhibited the good linearity with $r^{2}$ values over 0.9901 (Additional file 1: Table S7) at a certain concentration range in rat bile. From the data, we can see the developed method showed good linearity and sensibility in rat urine and bile.

\section{Plasma pharmacokinetics of circulating alkaloids and saponins in rats}

Table 1 summarized the PK parameters of circulating alkaloids (A1-A3), astragalosides (B2, B3) and ginsenosides (C1, C3, C5-C9, D2-D5) from intravenously dosed Kang-Ai injection. Figure 1a displayed the total plasma concentrations of the alkaloids over time, during and after a 30-min intravenous infusion of Kang-Ai injection in rats. Clearly, the plasma $C_{\max }$ value of matrine (A1) were determined after stopping the infusion for $30 \mathrm{~min}$. After the 30-min infusion, its plasma concentration increased with mean $t_{1 / 2}$ values of $4.33 \mathrm{~h}$. In addition, the $C_{\max }$ values of oxysophocarpine (A2) and oxymatrine (A3) were both measured just before stopping the infusion, following the declined plasma concentration with shorter $t_{1 / 2}$ values of 1.78 and $2.73 \mathrm{~h}$, respectively. The mean $V_{\mathrm{SS}}$ values of A1, $\mathbf{A} 2$ and $\mathbf{A} 3$ were 9.85, 25.20 and $220.11 \mathrm{~mL} / \mathrm{kg}$, respectively, suggesting these three alkaloids all had small $V_{\mathrm{d}}$ values. Besides, the $\mathrm{CL}_{\text {tot, } \mathrm{p}}$ values of $\mathbf{A} \mathbf{3}$ was $111.34 \mathrm{~mL} / \mathrm{h} / \mathrm{kg}$ and those of $\mathbf{A} \mathbf{1}$ and $\mathbf{A} \mathbf{2}$ were 1.58 and 12.31, respectively, indicating that $\mathbf{A} \mathbf{3}$ and $\mathbf{A 1}$, A2 had moderate and low total clearance, respectively. Furthermore, these circulating alkaloids (A1-A3) were not extensively bound with $f_{\mathrm{u} \text {-plasma }}$ values over $90 \%$.

The plasma concentration-time curves and PK parameters of astragaloside IV (B2) and III (B3) were shown in Fig. $1 \mathrm{~b}$ and Table 1, respectively. The plasma $C_{\max }$ $(2363.99 \mathrm{nmol} / \mathrm{L})$ and $\mathrm{AUC}_{0-\infty}(4523.65 \mathrm{nmol} / \mathrm{L} * \mathrm{~h})$ values of B2 were far higher than those of $\mathbf{B 3}$ with $C_{\max }$ and $\mathrm{AUC}_{0-\infty}$ values of $(54.75 \mathrm{nmol} / \mathrm{L})$ and $\mathrm{AUC}_{0-\infty}$ (34.29 nmol/L"h), due to the differences in the compounds' doses from the injection. Besides, the $t_{1 / 2}$ values of these two astragalosides were both small and less than 2 h. Similarly, B2 and B3 both exhibited the small $V_{\mathrm{d}}$ values $(<100 \mathrm{~mL} / \mathrm{kg})$, whereas $\mathbf{B 2}(28.39 \mathrm{~mL} / \mathrm{h} / \mathrm{kg})$ and $\mathbf{B 3}$ $(206.96 \mathrm{~mL} / \mathrm{h} / \mathrm{kg})$ had low and moderate total clearance, respectively. Protein binding assay demonstrated that B2 and B3 were extensively bound in rat plasma (nearly 90\%).

These circulating ginsenosides in plasma could be classified into two categories, protopanaxatriol (PPT) type ginsenosides (C1, C3, C5-C9) and protopanaxadiol $(P P D)$ type ginsenosides (D2-D5). Fig. 1c, d summarized the plasma concentrations of the PPT-type ginsenosides and $P P D$-type ginsenosides over time, respectively. It was notably shown that the $T_{\max }$ values of $P P T$-type ginsenosides were $30 \mathrm{~min}$, whereas those of PPD-type ginsenosides were measured after stopping infusion for $15 \mathrm{~min}$. In addition, the $C_{4 \mathrm{~h}}$ values of the PPT-type ginsenosides were very low, while those of the PPD-type ginsenosides still maintained a moderate plasma concentration. These suggested that the PPT-type ginsenosides possessed the longer MRT, and were eliminated more quickly than $P P D$-type ginsenosides. Another strong evidence was that $\mathrm{CL}_{\text {tot, }} \mathrm{p}$ values of PPT-type ginsenosides (72.70$255.80 \mathrm{~mL} / \mathrm{h} / \mathrm{kg}$ ) were approximately 10 times of those of PPT-type ginsenosides $(5.60-7.80 \mathrm{~mL} / \mathrm{h} / \mathrm{kg})$. Besides, except for ginsenoside Rg2 (C5) about the $t_{1 / 2}$ value of $9.77 \mathrm{~h}$, the PPT-type ginsenosides exhibited the shorter elimination half-lives values of i.e., $0.29-1.20 \mathrm{~h}$ for ginsenoside Rh1 (C1), Rg1 (C6), Rf (C7), Re (C9) and notoginsenoside R1 (C8), R2 (C3) than those of 4.12-7.22 $\mathrm{h}$ for ginsenosides Rd (D2), Rc (D3), Rb2 (D4) and Rb1 (D5). The $V_{\text {SS }}$ values of PPT-type ginsenosides and PPD-type ginsenosides were $50.05-791.61$ and $35.69-46.96 \mathrm{~mL} / \mathrm{kg}$, respectively, indicating that the PPT-type ginsenosides were more easily distributed in various body fluids and tissues than the PPD-type ginsenosides. Additionally, the $P P T$-type ginsenosides (C1, C3, C5-C9) were not significantly bound, whereas the PPD-type ginsenosides (D2D5) were obviously bound (around 99\%) in rat plasma.

Moreover, due to lack of the available authentic standards, virtual quantification of other main circulating herbal compounds in rat plasma, including baptifoline (A5), 9 $\alpha$-hydroxy-sophocarpine (A6), leontalbinine $\mathrm{N}$-oxide (A7), mamanine (A8), sophoranol (A9), were achieved using the calibration curve of an available reference standard of an analog that bore close structural similarity to the analyte. Figure 1e, Table 1 showed their mean plasma concentration-time curves and calculated relative PK parameters, respectively. The $C_{\max }$ values ranged from 202.37 to $421.27 \mathrm{nmol} / \mathrm{L}$, and the $T_{\max }$ values were all determined just before stopping the infusion. Besides, A7 exhibited the longest MRT $(7.94 \mathrm{~h})$ and $t_{1 / 2}$ $(5.73 \mathrm{~h})$ values among these five alkaloids. The small $V_{\mathrm{SS}}$ (16.89-150.70 mL/kg) and $\mathrm{CL}_{\text {tot, } \mathrm{p}}(7.93-56.05 \mathrm{~mL} / \mathrm{h} / \mathrm{kg})$ values indicated that these alkaloids were slowly distributed in various body fluids and tissues, and slowly cleared from the systemic circulation.

To better demonstrate the systemic exposure in rat plasma, the comparison of the $\mathrm{AUC}_{0-12 \mathrm{~h}}$ values of circulation alkaloids and saponins were displayed in Fig. 2a. Obviously, oxymatrine (A3) exhibited the most abundant exposure with $502.71 \mu \mathrm{mol} / \mathrm{L} * \mathrm{~h}$, due to the most abundant dose level from Kang-Ai injection. Additionally, A1, B2, D3, D2, D5, C6, C9, A7, D4, C7, A8, A6, A9, A5, A2, C5, C8, in descending order, exhibited substantially higher total levels of plasma exposure with the $\mathrm{AUC}_{0-12 \mathrm{~h}}$ 


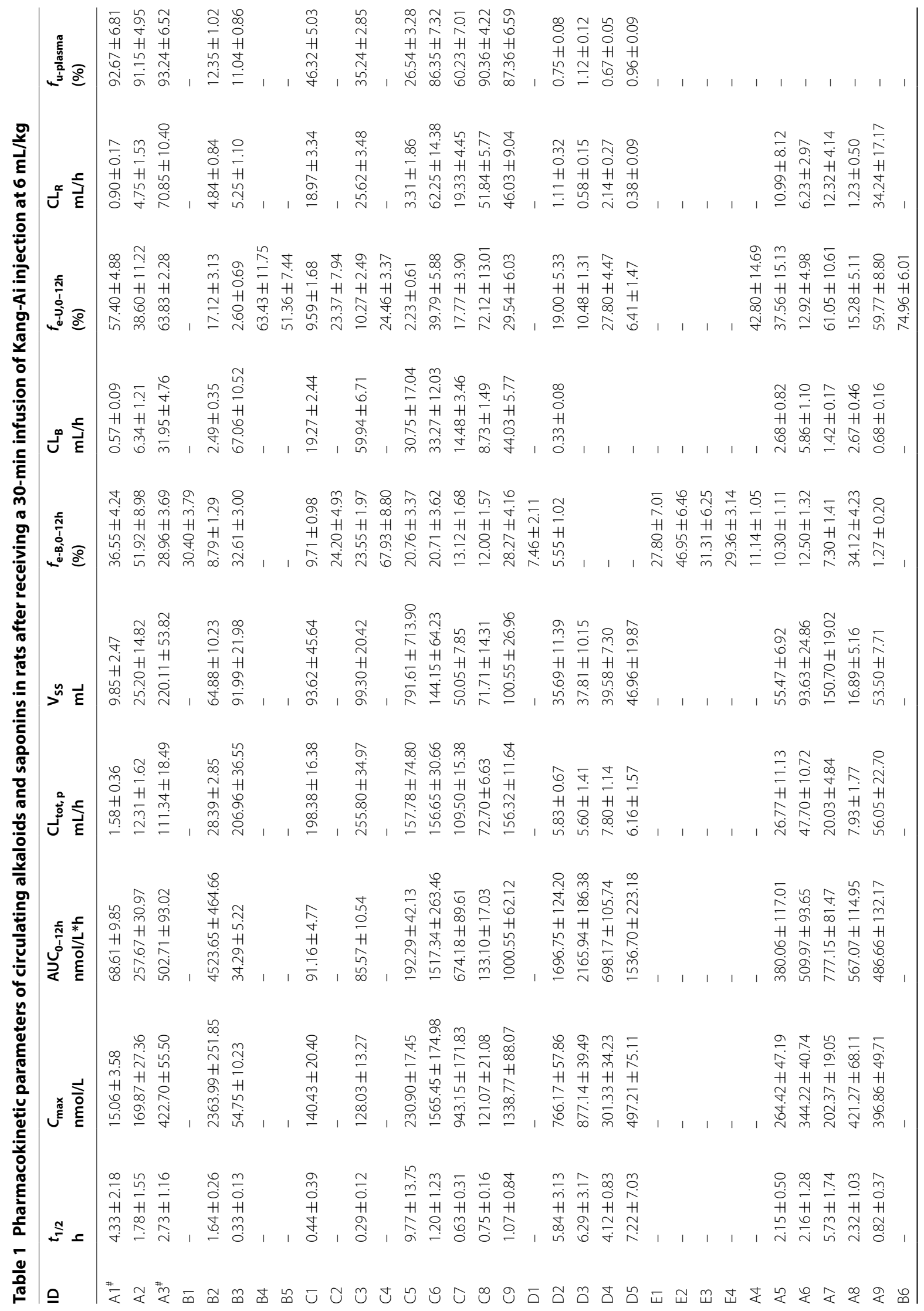




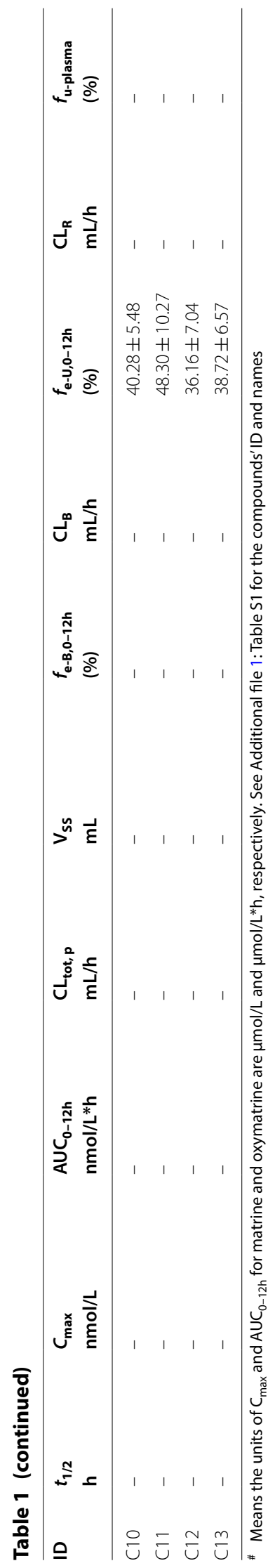




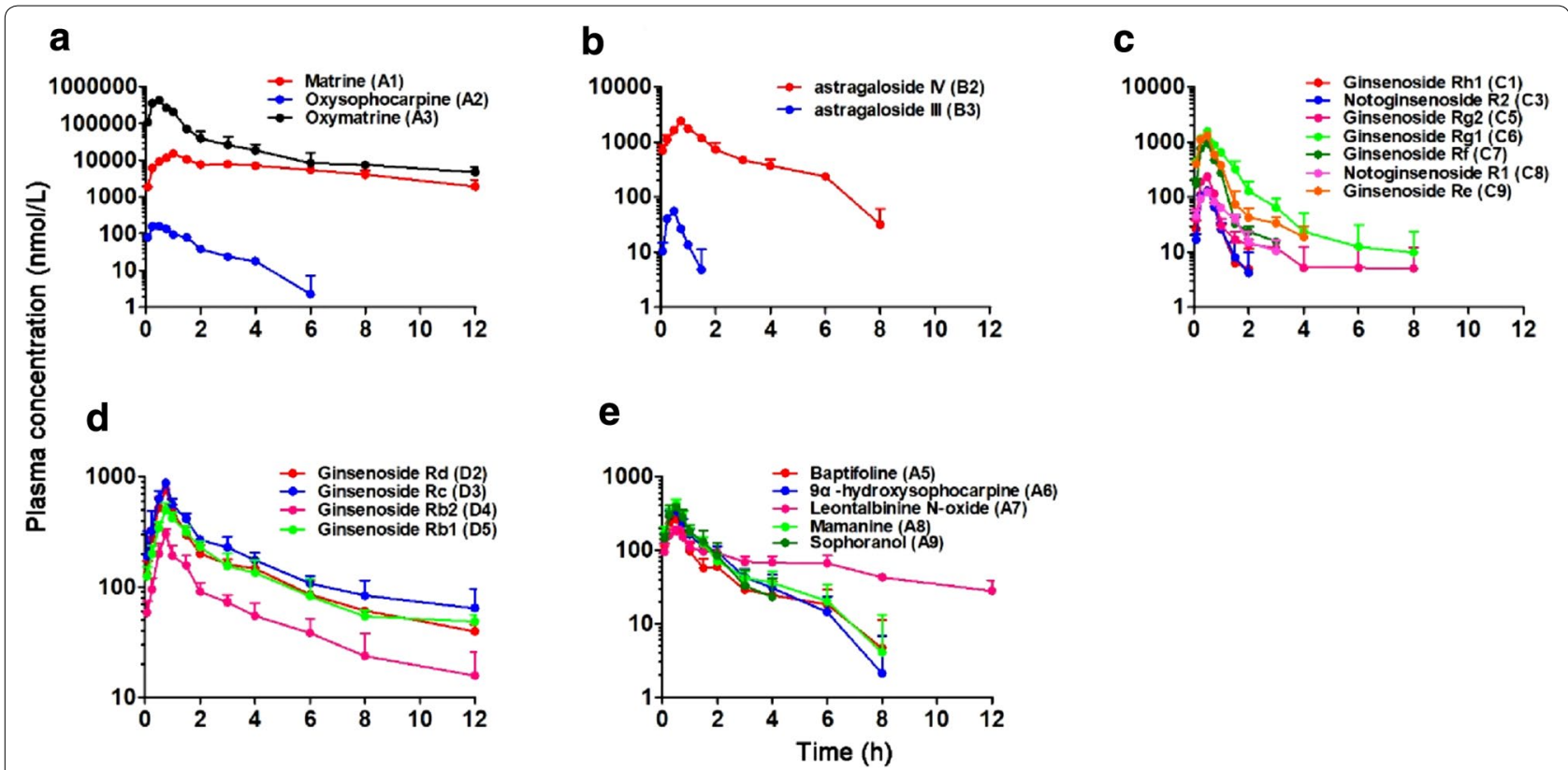

Fig. 1 Mean plasma pharmacokinetics of three abundant alkaloids (a), two astragalosides (b), seven PPT-type ginsenosides (c), four PPD-type ginsenosides (d) and other five minor alkaloids (e) over the time after receiving an intravenous 30-min infusion of Kang-Ai injection in rats (6 mL/ kg). See Additional file 1: Table S1 for the compounds' ID and names

values over $100 \mathrm{nmol} / \mathrm{L}$ "h, whereas the levels of plasma exposure to other compounds (B3, C1, C3) were quite low, due to their low compound dose from Kang-Ai injection.

\section{Urinary and biliary excretion of circulating alkaloids and saponins in rats}

Table 1 summarized the urinary and biliary excretion parameters of circulating alkaloids and saponins. Meanwhile, Fig. 2b, c showed their cumulative excretion amounts in urine and bile, respectively. For the circulating alkaloids (A1-A3), the renal and hepatobiliary excretion of the unchanged compounds represented the major routes of elimination (Table 1). This was attributed that A1 (57.40\%), A2 (38.60\%) and A3 (63.83\%) exhibited an extraordinarily high $f_{\mathrm{e}-\mathrm{U}, 0-12 \mathrm{~h}}$ values, the sum of which with the $f_{\mathrm{e}-\mathrm{B}, 0-12 \mathrm{~h}}$ values $(36.55 \%, 51.92 \%, 28.96 \%$, respectively) exceeded $90 \%$ of individual dose levels.

Astragaloside III (B3) was eliminated more extensively via hepatobiliary excretion $\left(f_{\mathrm{e}-\mathrm{B}}, 67.06 \%\right)$ than renal excretion $\left(f_{\mathrm{e}-\mathrm{U}}, 5.25 \%\right)$ (Table 1). Unlike for B3, renal excretion was a minor elimination pathway for astragaloside IV (B2) $\left(f_{\mathrm{e}-\mathrm{U}}, 4.84 \%\right)$, and the same fact was that the fraction of dosed B2 was excreted into bile $\left(f_{\mathrm{e}-\mathrm{B}}\right)$ about $2.49 \%$.

The PPT-type ginsenosides (C1-C9), except ginsenoside Rg2 (C5), all exhibited the large $f_{\mathrm{e}-\mathrm{U}}$ values (9.59$72.17 \%$ ) (Table 1), suggesting that the renal excretion was the predominant elimination route responsible for their systemic clearance. Similarly, the PPD-type ginsenosides (D2-D5), exhibited moderate $f_{\mathrm{e}-\mathrm{U}}$ values $(6.41-27.8 \%)$, which was around a half of the values of PPT-type ginsenosides. In addition, in rat bile, the PPT-type ginsenosides were the most abundant herbal compounds with $f_{\mathrm{e}-\mathrm{B}}$ values of $9.71-67.93 \%$, whereas only two PPD-type ginsenosides, ginsenoside F2 (D1) and Rd (D2) could be detected and displayed the small $f_{\mathrm{e}-\mathrm{B}}$ values of $7.46 \%$ and $5.55 \%$, respectively. Consistent with the inter-compound differences in $\mathrm{CL}_{\text {tot, } p}$ values, the $\mathrm{CL}_{\mathrm{R}}$ and $\mathrm{CL}_{\mathrm{B}}$ values for these PPT-type ginsenosides (C1-C9) were significantly greater than those respect values for the PPD-type ginsenosides (D1-D5).

Moreover, other alkaloids (A4-A9) were eliminated more extensively unchanged via the renal excretion $\left(f_{\mathrm{e}}\right.$ $\mathrm{U}, 12.92-61.05 \%)$ than the hepatobiliary excretion $\left(f_{\mathrm{e}-\mathrm{B}}\right.$, 1.27-34.12\%) (Table 1). The $\mathrm{CL}_{\mathrm{R}}$ and $\mathrm{CL}_{\mathrm{B}}$ values for the alkaloids (A4-A9) kept in line with their $\mathrm{CL}_{\text {tot, } \mathrm{p}}$ values. Another astragaloside, astragaloside VII (B6) was excreted $74.96 \%$ of the dose derived from Kang-Ai injection to urine. Another four PPT-type ginsenosides, ginsenosides R1 (C10), Re4 (C11), notoginsenoside R3 (C12) and 20-gluco-ginsenoside Rf (C13) were all subjected to be eliminated by the renal excretion with $f_{\mathrm{e}-\mathrm{U}}$ values of $36.16-48.30 \%$, whereas their hepatobiliary excretion appeared to be negligible. 

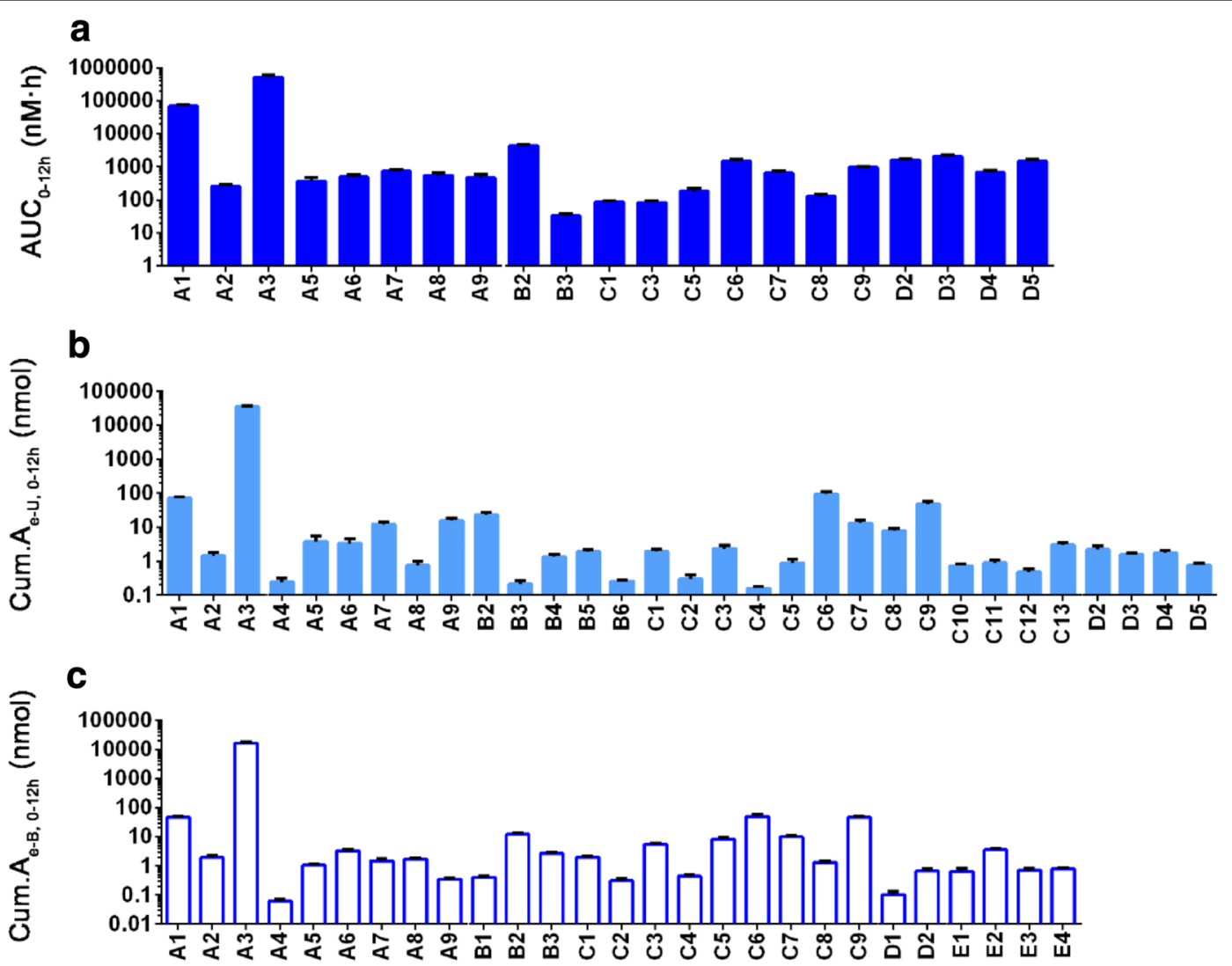

\section{Circulating alkaloids and saponins}

Fig. 2 Systemic exposure in plasma (a), renal excretion in urine (b) and hepatobiliary excretion in bile (c) of circulating alkaloids, astragalosides and ginsenosides after a 30-min intravenous infusion of Kang-Ai injection to rats $(6 \mathrm{~mL} / \mathrm{kg})$. See Additional file 1: Table S1 for the compounds' ID and names

\section{Inhibitory effects of Kang-Ai injection and herbal} compounds towards each CYP and UGT isozyme

Kang-Ai injection showed inhibitory effects towards CYP2A6, 2E1 and 3A4 with residual activity of 73.63\%, $70.28 \%$ and $56.92 \%$, respectively (Fig. 3a). Meanwhile, Kang-Ai injection almost exhibited no inhibitory effects against other CYP and UGT enzymes (Fig. 3a). Based on Table 1, matrine (A1), oxymatrine (A3), astragaloside IV (B2), ginsenosides Rg1 (C6), Rf (C7), Re (C9), $\mathrm{Rd}$ (D2), Rc (D3), Rb1 (D5) exhibited the $\mathrm{C}_{\max }$ values about or over $0.5 \mu \mathrm{mol} / \mathrm{L}$ after intravenous Kang-Ai injection. Therefore, these nine major circulating compounds were selected to evaluate the inhibitory effects toward several common CYP and UGT enzymes. A1 $(100 \mu \mathrm{M})$ displayed inhibitory effects against CYP3A4 (66.63\%) (Additional file 1: Fig. S5A), whereas A3 $(100 \mu \mathrm{M})$ could inhibit the metabolic activity of CYP2A6 (77.63\%), 2E1 (80.28\%), and 3A4 (64.92\%) (Fig. 3b). In addition, B2 $(100 \mu \mathrm{M})$ exhibited inhibitory activity towards CYP2C9 (52.36\%) (Fig. 3c). Besides,
PPT-type ginsenosides, C6 (100 $\mu \mathrm{M})$ (Fig. 3d) and C9 $(100 \mu \mathrm{M})$ (Additional file 1: Fig. S5C) mainly inhibited the function of UGT1A1 (53.26\%) and CYP2C8 (30.25\%), respectively, while almost no changes were observed for C7 (100 $\mu \mathrm{M})$ against these CYP and UGT enzymes (Additional file 1: Fig. S5B). Furthermore, D2 $(100 \mu \mathrm{M})$ exhibited inhibition on the metabolic activity of CYP2B6 (30.36\%) and 3A4 (42.36\%) (Additional file 1: Fig. S5D), whereas D3 $(100 \mu \mathrm{M})$ (Fig. 3e) and D5 $(100 \mu \mathrm{M})$ (Additional file 1: Fig. S5E) showed inhibitory effects on UGT1A9 (30.26\%) and CYP2C9 (15.36\%), respectively.

Moreover, the inhibition data (Fig. 4) were fit to log (I) and normalized response equations to obtain the $\mathrm{IC}_{50}$ values. The inhibition trend of the abundant compounds towards these CYP and UGT enzymes showed in a dose dependent manner, and their $\mathrm{IC}_{50}$ values were 65.00 (B2 towards CYP2C9), 92.21 (C6 against UGT1A1), 24.03 (C9 towards CYP2C8), 20.19 (D2 against CYP2B6), 40.96 (D2 towards CYP3A4), 28.96 (D3 against UGT1A9) and 
$8.81 \mu \mathrm{M}$ (D5 towards CYP2C9), respectively (Additional file 1: Table S8). The inhibition mechanism was further evaluated based on three inhibition kinetic models.

\section{Analysis of inhibition mechanism}

The AIC and SC values (Table 2) were obtained after the inhibition data were modeled by three conventional inhibition equation (i.e., competitive, noncompetitive and mixed-type). Based on the smallest AIC and SC values principle, $\mathbf{C} 6$ displayed competitive inhibition against UGT1A1, while C9 exhibited mixed-type inhibition against CYP2C8. Noncompetitive inhibition kinetics were observed for B2 against CYP2C9, D2 towards CYP2B6 and 3A4, D3 against UGT1A9 and D5 towards CYP2C9.

Furthermore, the Dixon plots for B2 against CYP2C9 (Fig. 5a), C6 towards UGT1A1 (Fig. 5b), C9 against CYP2C8 (Fig. 5c), D2 towards CYP2B6 (Fig. 5d) and 3A4 (Fig. 5e), D3 against UGT1A9 (Fig. 5f) and D5 towards CYP2C9 (Fig. 5g) also provided strong evidences to support this judgment about the inhibition mechanism. Their respective $K_{i}$ values were $55.8,140.6,13.3$, 37.2,
32.8, 22.3 and $16.1 \mu \mathrm{M}$ (Table 2). These findings suggested that the major circulating compounds have weak or no inhibition on the metabolic activity of common CYP and UGT enzymes with $\mathrm{K}_{\mathrm{i}}$ values all over $10 \mu \mathrm{M}$.

\section{Potential DDI risks}

Based on the $[\mathrm{I}] / \mathrm{K}_{\mathrm{i}}$ values in vitro, potential DDI risks in vivo could be predicted $[27,28]$. Traditionally, [I]/ $\mathrm{Ki}<0.1$, low possibility DDI; $1>[\mathrm{I}] / \mathrm{Ki}>0.1$, medium possibility DDI; [I]/Ki $>1$, high possibility DDI [18]. In this study, $[\mathrm{I}] / \mathrm{K}_{\mathrm{i}}$ values for these herbal compounds were all no more than 0.1 (Table 2). Therefore, it seemed impossible to trigger DDI when Kang-Ai injection were co-administrated with clinical drugs which were metabolized by these enzymes.

\section{Discussion}

Bioactive compounds with adequate abundance in herbal injection and favorable PK properties are mostly believed to be responsible for the pharmacological effects and therapeutic efficacy [4]. In the current study, alkaloids and saponins derived from Kang-Ai injection are two
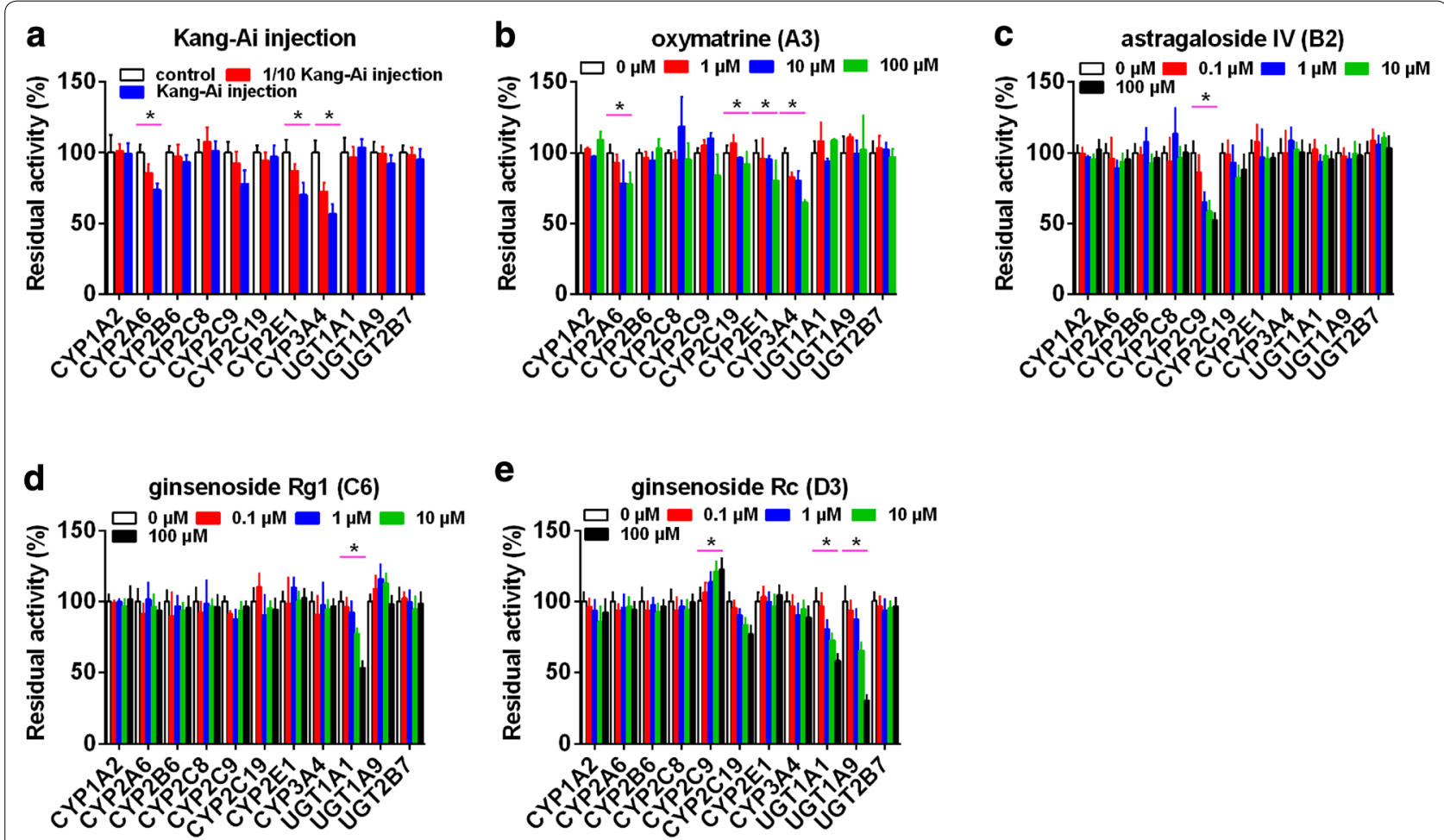

Fig. 3 The effects of several circulating compounds towards eight expressed CYP isozymes and three recombinant UGT enzymes. a Kang-Ai injection, $\mathbf{b}$ oxymatrine, $\mathbf{c}$ astragaloside IV, $\mathbf{d}$ ginsenosides $\mathrm{Rg} 1$, and $\mathbf{e}$ ginsenoside $\mathrm{Rc}$; The probe substrates were incubated at $37^{\circ} \mathrm{C}$ in the absence (control, $0 \mu \mathrm{M}$ ) and presence of tested mixture and compounds (Kang-Ai injection and samples that Kang-Ai injection was diluted 10 times; 1, 10, and $100 \mu \mathrm{M}$ for oxymatrine; 0.1, 1, 10 and $100 \mu \mathrm{M}$ for astragalosides and ginsenosides). Data represent the mean \pm standard deviation of triplicate, ${ }^{*}$ compared with those of control, * $p<0.05$ ) 

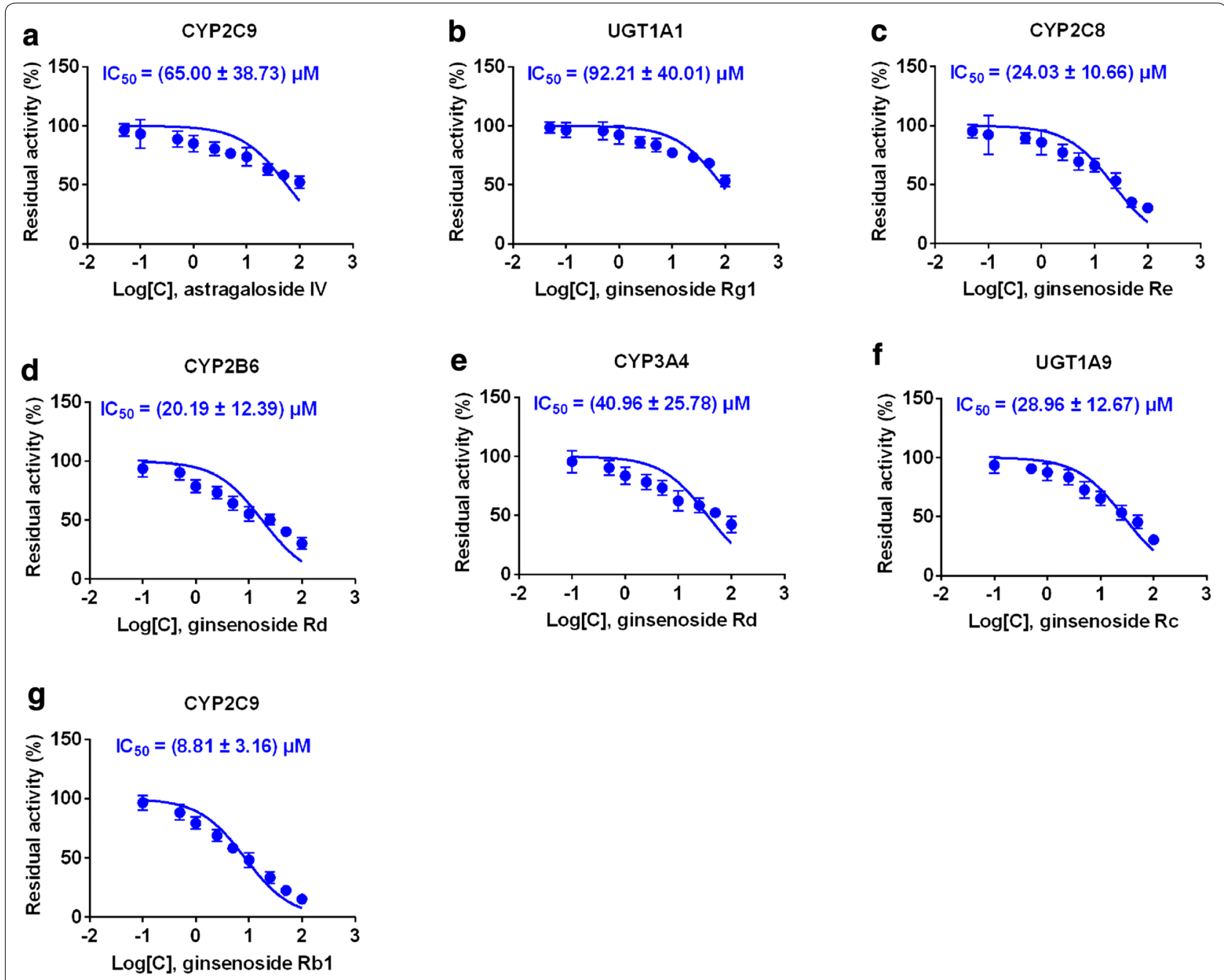

Fig. 4 The IC50 values of astragaloside IV against CYP2C9 (a), ginsenoside Rg1 against UGT1A1 (b), ginsenoside Re towards CYP2C8 (c), ginsenoside Rd towards CYP2B6 (d) and CYP3A4 (e), ginsenoside Rc against UGT1A9 (f), and ginsenoside Rb1 against CYP2C9 (g). The data were fit to log (concentration) and normalized response equations. Each data point represented the mean value \pm the S.D. of triplicate determination

classes of bioactive compounds which are likely responsible for the adjuvant therapy of many cancers (Additional file 1: Table S1) [6, 8]. After dosing, oxymatrine (A3), matrine (A1), astragaloside IV (B2), ginsenosides Rc (D3), Rd (D2), Rb1 (D5), Rg1 (C6) and Re (C9), all exhibited considerably high levels of systemic exposure (over $1000 \mathrm{nmol} / \mathrm{L} * \mathrm{~h}$ ) (Fig. 2a), indicating that these circulating compounds are worth special consideration in the pharmacological research. Besides, the abundant exposure of A1 may be attributed the metabolism of A3 by CYP3As [29]. In addition, the considerable systemic exposure for A3, A1, D3, D2 and D5, seemed to be correlated with the significantly longer $t_{1 / 2}$ values of $2.73-7.22 \mathrm{~h}$ than those of B2, C6 and C9 (1.07-1.64 h) (Table 1). This phenomenon that long elimination half-life could significantly counterbalance influence on systemic exposure to herbal compounds with poor bioavailability has been proved previously [30].

Except the systemic exposure of major circulating compounds, we also investigate their elimination in the current study (Table 1). After dosing Kang-Ai injection, oxymatrine (A3) and matrine (A1) mainly involved in the renal and hepatobiliary excretion of the parent compounds (over 90\%), of which parts of circulated A1 was originated from the metabolism of A3 by CYP3A4 [11]. Only about $25 \%$ of dosed astragaloside IV (B2) were detected as parent compound in urine and bile, and this was probably attributed to the de-glycosylation of $\mathbf{B 2}$ to its sapogenin [15]. Additionally, ginsenosides Rg1 (C6) and $\operatorname{Re}(\mathbf{C 9})$ were eliminated mainly to urine and bile 
Table 2 Inhibition parameters of circulating alkaloids and saponins against several CYP and UGT isozymes

\begin{tabular}{|c|c|c|c|c|c|c|c|c|c|}
\hline Compounds & Isozymes & Inhibition type & $\mathrm{K}_{\mathrm{i}}(\mu \mathrm{M})$ & $a$ & $R^{2}$ & AICs & SCs & Selection & $\mathrm{C}_{\max } / \mathrm{K}_{\mathrm{i}}$ \\
\hline \multirow[t]{3}{*}{ Astragaloside IV (B2) } & CYP2C9 & Competitive & $17.4 \pm 3.7$ & - & 0.9480 & -113.3 & -110.3 & & \\
\hline & & Noncompetitive & $55.8 \pm 5.3$ & - & 0.9708 & -124.8 & -121.8 & $\sqrt{ }$ & 0.04 \\
\hline & & Mixed-type & $45.0 \pm 16.6$ & $1.5 \pm 1.0$ & 0.9713 & -123.1 & -119.2 & & \\
\hline \multirow[t]{3}{*}{ Ginsenoside Rg1 (C6) } & UGT1A1 & Competitive & $140.6 \pm 10.5$ & - & 0.9952 & 176.4 & 179.4 & $\sqrt{ }$ & 0.01 \\
\hline & & Noncompetitive & $187.2 \pm 12.7$ & - & 0.9943 & 179.8 & 182.8 & & \\
\hline & & Mixed-type & $147.3 \pm 29.9$ & $8.3 \pm 37.2$ & 0.9952 & 178.7 & 182.7 & & \\
\hline \multirow[t]{3}{*}{ Ginsenoside Re (C9) } & CYP2C8 & Competitive & $8.2 \pm 1.1$ & - & 0.9790 & -121.3 & -118.3 & & \\
\hline & & Noncompetitive & $26.5 \pm 2.2$ & - & 0.9793 & -121.6 & -118.6 & & \\
\hline & & Mixed-type & $13.3 \pm 2.9$ & $4.2 \pm 2.3$ & 0.9862 & -127.8 & -123.8 & $\sqrt{ }$ & 0.1 \\
\hline \multirow[t]{3}{*}{ Ginsenoside Rd (D2) } & CYP2B6 & Competitive & $10.2 \pm 2.1$ & - & 0.9484 & 19.4 & 22.4 & & \\
\hline & & Noncompetitive & $37.2 \pm 2.3$ & - & 0.9877 & -9.2 & -6.2 & $\sqrt{ }$ & 0.02 \\
\hline & & Mixed-type & $36.3 \pm 10.2$ & $1.0 \pm 0.4$ & 0.9877 & -7.2 & -3.2 & & \\
\hline \multirow[t]{3}{*}{ Ginsenoside Rd (D2) } & CYP3A4 & Competitive & $8.7 \pm 2.3$ & - & 0.9226 & -31.9 & -28.9 & & \\
\hline & & Noncompetitive & $32.8 \pm 2.2$ & - & 0.9846 & -64.2 & -61.2 & $\sqrt{ }$ & 0.02 \\
\hline & & Mixed-type & $44.2 \pm 16.2$ & $0.6 \pm 0.3$ & 0.9854 & -63.3 & -59.3 & & \\
\hline \multirow[t]{3}{*}{ Ginsenoside Rc (D3) } & UGT1A9 & Competitive & $8.9 \pm 1.5$ & - & 0.9634 & 122.8 & 125.8 & & \\
\hline & & Noncompetitive & $22.3 \pm 1.4$ & - & 0.9877 & 100.9 & 103.9 & $\sqrt{ }$ & 0.04 \\
\hline & & Mixed-type & $27.0 \pm 7.5$ & $0.7 \pm 0.3$ & 0.9881 & 102.3 & 106.3 & & \\
\hline \multirow[t]{3}{*}{ Ginsenoside Rb1 (D5) } & CYP2C9 & Competitive & $8.2 \pm 1.0$ & - & 0.9812 & -115.9 & -112.9 & & \\
\hline & & Noncompetitive & $16.1 \pm 0.9$ & - & 0.9921 & -133.2 & -130.3 & $\sqrt{ }$ & 0.03 \\
\hline & & Mixed-type & $18.8 \pm 4.3$ & $0.7 \pm 0.3$ & 0.9923 & -131.8 & -127.9 & & \\
\hline
\end{tabular}

Data represent the mean \pm standard deviation of triplicate

$\mathrm{C}_{\max } / \mathrm{K}_{\mathrm{i}}$ also named $[\mathrm{l}] / \mathrm{K}_{\mathrm{i}}$ standard ([l]/Ki $<0.1$, low possibility DDI; $1>[\mathrm{l}] / \mathrm{Ki}>0.1$, medium possibility DDI; [l]/Ki $>1$, high possibility DDI)

(nearly 60\%), whereas it proved that approximately $25 \%$ of ginsenoside Rd (D2) was excreted via renal and hepatobiliary elimination. Ginsenosides Rc (D3) and Rb1 (D5) were not detected in bile, and mainly were eliminated through renal excretion. Except the in vivo metabolism, i.e., oxidation and de-glycosylation [16], the delivery to the rat tissues of circulating herbal compounds through blood flow were also an important factor to determine the systemic exposure and excretion of circulating compounds [31]. The $\mathrm{V}_{\mathrm{SS}}$ values of dosed A3, C6 and C9 were $100.55-220.11 \mathrm{~mL} / \mathrm{kg}$, which were near the rat extracellular volume $(300 \mathrm{~mL} / \mathrm{kg})$ [32], indicating that these herbal compounds could be distributed evenly in various body tissues, except several barriered tissues. However, this elimination route warrant future investigation.

In addition, the obtained PK parameters (Table 1) also could enable us to compare the plasma concentrations of the alkaloids and saponins with the reported effective concentrations $\left(\mathrm{IC}_{50}\right.$ or $\mathrm{EC}_{50}$ values) for pharmacological activities. Oxymatrine (A3) exhibited the most abundant concentration $(422.70 \mu \mathrm{mol} / \mathrm{L})$ after dosing Kang-Ai injection, which is still obviously below the reported effective concentrations $(473.48-3787.84 \mu \mathrm{mol} / \mathrm{L})$ for several tumor cell lines, including HepG2, MCF-7, HeLa, A549 cells [33]. However, for SW-620, BGC823, A375 cells, A3 is sufficiently effective to inhibit the tumor cell viability $(0.10-40.00 \mu \mathrm{mol} / \mathrm{L})$ [33]. Similar results were obtained for matrine (A1) and its derivatives [34], astragaloside IV (B2) [35], and several main ginsenosides [36]. Although the low plasma concentrations do not result in the ineffectiveness of Kang-Ai injection for the therapeutic effects, they may limit the contribution of bioactive compounds to the current adjuvant therapy. How to achieve the higher plasma concentrations is an important idea to develop new and superior adjuvant agents derived from the current Kang-Ai injection.

Kang-Ai injection are the combinations of two herbs and Kushensu, of which complex chemical compositions increased the DDI risks. Usually, the induction and inhibition of CYP and UGT enzymes can facilitate the metabolism and elimination of their drug substrates and caused unexpected adverse effect. Previously, oxymatrine (A3) was subjected to be metabolized to matrine (A1) rapidly in pooled HLM $\left(K_{\mathrm{m}}=220.78 \mu \mathrm{M}\right)$ and HIM $\left(K_{\mathrm{m}}=150.22 \mu \mathrm{M}\right)$, and CYP3A4 $\left(K_{\mathrm{m}}=341.35 \mu \mathrm{M}\right)$ isozyme greatly contributed to this transformation [37]. However, A1 could not be extensively metabolized by CYPs and UGTs [38]. In addition, considering that most of clinical drugs or endogenous compounds are metabolized or eliminated by CYP1A2 (9\%), 2A6, 2B6 (2\%), 


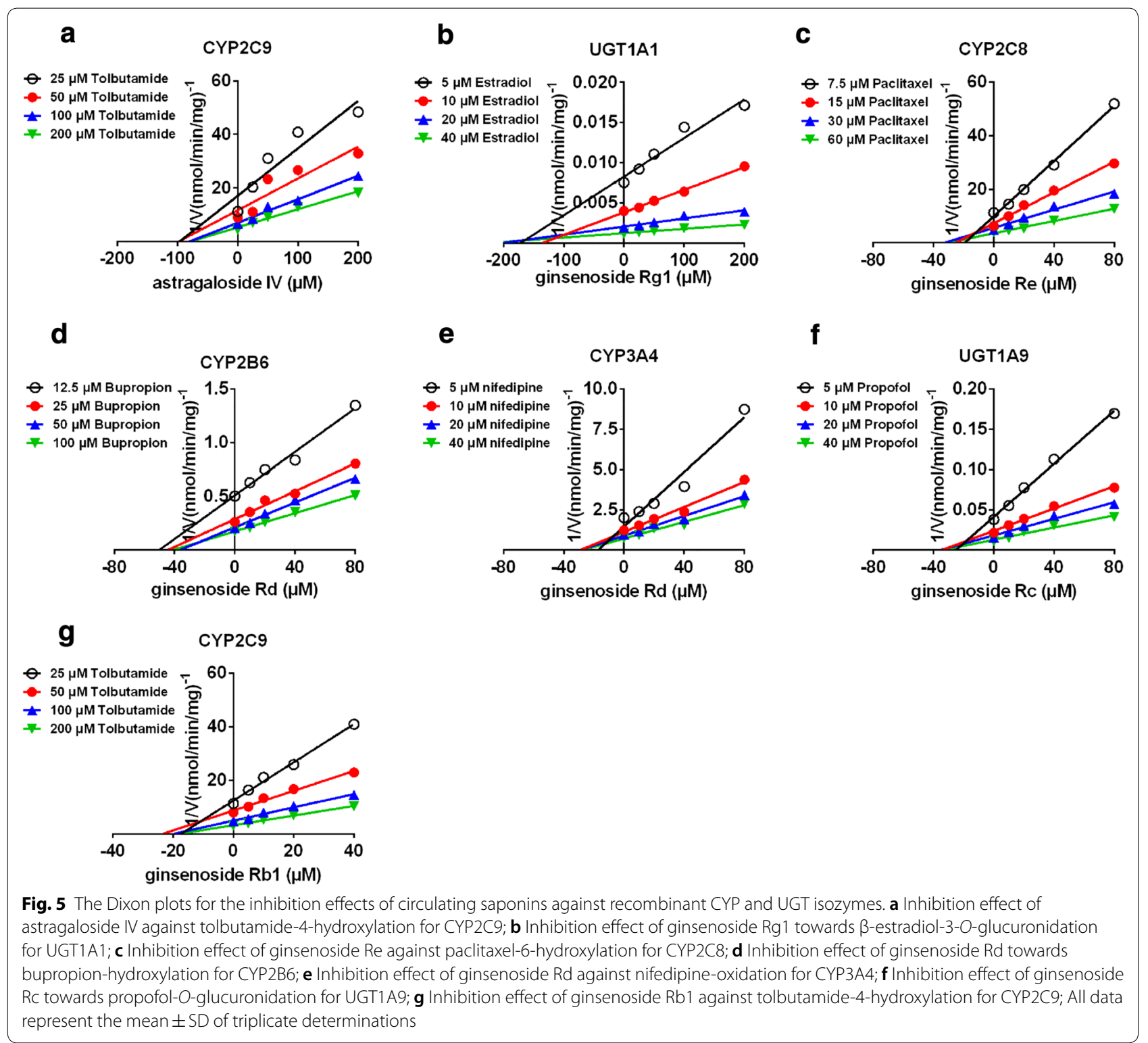

2C8, 2 C9 (16\%), 2C19 (12\%), 2E1 (2\%), 3A4 (46\%), and UGT1A1 (15\%), 1A9, 2 B7 (35\%) [39, 40], we focused on the inhibitory effects of herbal compounds derived from Kang-Ai injection against these enzymes above.

Our results showed that Kang-Ai injection demonstrated weak inhibitory effects against CYP2A6, 2E1 and 3A4 (Fig. 3a), which could be mainly attributed to the contribution of oxymatrine. This is because the content level of oxymatrine in Kang-Ai injection $(37731.41 \mu \mathrm{M})$ is far higher than those of other herbal compounds (0.23$158.95 \mu \mathrm{M}$ ) (Additional file 1: Table S1) [9]. Furthermore, it was notable that the de-glycosylation of astragaloside IV (B2) to sapogenin-cycloastragenol strongly increased the inhibitory effects towards UGT1A8 $\left(K_{\mathrm{i}}=0.034 \mu \mathrm{M}\right)$ and 2B7 $\left(K_{\mathrm{i}}=20.98 \mu \mathrm{M}\right)$ [15]. Interestingly, naturally occurring alkaloids and ginsenosides, including A1, A3, ginsenosides Rg1 (C6), Rf (C7), Re (C9), Rd (D2), Rc (D3) and Rb1 (D5), were unlikely to cause clinically relevant DDI mediated via the induction or inhibition of most CYPs or UGTs involving in drug metabolism in vivo, which agreed with previous study [41].

Identification of the induction or inhibition of the abundant circulating alkaloids and saponins towards uptake and efflux transporters was another significant risk factor for the DDI. In clinics, oxymatrine (A3) could interfere with hOCT1-mediated hepatic uptake and renal elimination, and intestinal absorption by hOCT3, whereas matrine (A1) only had the potential to decrease 
hOCT3 expressed in enterocytes [42]. Besides, A1 and A3 did not demonstrate the inhibition of P-gp in vitro and in vivo [43]. Additionally, it was reported that astragaloside IV (B2) could induce the protein levels of P-gp, MRP1, MRP3, but produced the inhibitory effects on BCRP [44]. In contrast, in MDR1-MDCKII cells, B2 only exhibited weak inhibition activity of $8.22 \%$ when treated with $100 \mu \mathrm{M}$ [43]. Recently, several scholars got the fact that B2 could induce the upregulation of P-gp and BCRP, and might be a potential substrate of P-gp [45]. For ginsenosides, ginsenoside Rg1 (C6) was potential inhibitor of NTCP $\left(\mathrm{IC}_{50}=50.49 \mu \mathrm{M}\right)$, whereas ginsenoside Re (C9) was potential substrates of NTCP [46]. Further, DDI between ginsenosides Rb1 (D5), Rc (D3), Rd (D2) and methotrexate (a probe substrate for Mrp2) might occur owing to the decrease in the mRNA and protein levels of Mrp2 [47]. In addition, D5, D3, D2 and D9 were predicted to possess the high potential for human OATP1B3-mediated drug interactions [16]. Moreover, $P P T$ and $P P D$, the aglycones of PPT-type ginsenosides and $P P D$-type ginsenosides, respectively, were potent P-gp inhibitors, suggesting the unpredictable interactions when ginsenosides were co-administered with the P-gp substrate drugs [48]. The interactions of complex circulating compounds and clinical drugs based on transporters may be one of the important compatibility mechanisms for Kang-Ai injection.

In clinical consumption, the adverse drug reactions (ADR) derived from herbal injections, should be gaining more attention. It was essential to evaluate the objectivity of ADR, develop a reasonable application strategy, and ensure the security and validity of Kang-Ai injection. Data and information obtained from PK studies on the circulating alkaloids and saponins could help understand the complex action of herbal injections, and predict a variety of events related to the efficacy and toxicity of herbal injections. In Kang-Ai injection, the most abundant compounds, oxymatrine (A3) and matrine (A1) both could cause the hepatic toxicities, of which the toxicity of A1 was greater than that of A3 [49]. The elevated metabolism of $\mathbf{A} 3$ by CYP3A4 may result in more severe liver damage. Therefore, the clarification of the PK studies could be helpful in deciphering the ADR and hepatorenal toxicity of herbal injections.

\section{Conclusion}

Herein, nine alkaloids, six astragalosides and twentytwo ginsenosides were the main circulating compounds after dosing Kang-Ai injection. Oxymatrine (A3), matrine (A1), astragaloside IV (B2), ginsenosides Rc (D3), Rd (D2), Rb1 (D5), Rg1 (C6), Re (C9), Rf (C7), and Rb2 (D4) exhibited high exposure in plasma. Renal and hepatobiliary excretion were their major elimination pathways. In addition, PPD-type ginsenosides (D2-D5) were extensively bound with $f_{\mathrm{u}}$ plasma values approximately $1 \%$, whereas alkaloids and PPT-type ginsenosides (C6, C7, C9) displayed higher $f_{\text {u-plasma }}$ values (60.23-93.24\%). Furthermore, A1, A3, B2, C6, C7, C9, D2, D3 and D5 displayed weak inhibition or no inhibition towards several common CYP and UGT enzymes with $\mathrm{IC}_{50}$ values ranging from 8.81 to $92.21 \mu \mathrm{M}$, suggesting negligible DDI risk. Taken together, the current PK and excretion information gained here could serve as a crucial step in understanding the roles of circulating alkaloids, astragalosides and ginsenosides in therapeutic actions of Kang-Ai injection. These results here will also inform the negligible DDI risks in clinical use of Kang-Ai injection.

\section{Supplementary information}

Supplementary information accompanies this paper at https://doi. org/10.1186/s13020-020-00349-3.

\footnotetext{
Additional file 1: Table S1. Information of unchanged compounds in rats after a 30 min-infusion of Kang-Ai injection $(6 \mathrm{~mL} / \mathrm{kg}$ ) by UHPLC/Q-TOFMS. Table S2. Linear correlation parameters, limits of detection (LODs) and lower limits of quantification (LLOQs) of each circulating compound in rat plasma after a 30 min-infusion of Kang-Ai injection ( $6 \mathrm{~mL} / \mathrm{kg})$. Table S3. Relative standard deviation (RSD) values of intra-day and inter-day precisions, and of sixteen compounds in rat plasma after a 30 min-infusion of Kang-Ai injection ( $6 \mathrm{~mL} / \mathrm{kg}$ ). Table $\mathbf{S 4}$. Matrix effect and extraction recovery of each compound and two internal standard (IS) in rat plasma after a 30 min-infusion of Kang-Ai injection ( $6 \mathrm{~mL} / \mathrm{kg}$ ). Table S5. Stability results of each compound and internal standard in rat plasma after a 30 mininfusion of Kang-Ai injection ( $6 \mathrm{~mL} / \mathrm{kg}$ ). Table S6. Linear correlation parameters, LLOQs of twenty analytes in rat urine after a 30 min-infusion of Kang-Ai injection ( $6 \mathrm{~mL} / \mathrm{kg}$ ). Table S7. Linear correlation parameters, LLOQs of twenty-one analytes in rat bile after a 30 min-infusion of Kang-Ai injection $(6 \mathrm{~mL} / \mathrm{kg})$. Table $\mathbf{S 8}$. The $\mathrm{IC}_{50}$ values of six circulating alkaloids and saponins towards several recombinant CYP and UGT isozymes. Data represent the mean \pm standard deviation of triplicate. Fig S1. Effects of different incubation conditions on the metabolism of mephenytoin by human CYP2C19 (mean $\pm S D, n=3$ ). (A) Tris buffer concentration; (B) $\mathrm{pH}$ values; (C) $\mathrm{MgCl}_{2}$ concentration; (D) NADPH concentration; (E) incubation time; (F) protein concentration. Fig S2. Effects of different incubation conditions on the metabolism of estradiol by UGT1A1. (A) Tris buffer concentration; (B) $\mathrm{pH}$ values; (C) $\mathrm{MgCl}_{2}$ concentration; (D) UDPGA concentration; (E) incubation time; $(F)$ protein concentration. Data represent the mean \pm standard deviation of triplicate. Fig S3. Chemical structures (A) and content levels (B) of circulating alkaloids, astragalosides and ginsenosides in rats after intravenous Kang-Ai injection $(6 \mathrm{~mL} / \mathrm{kg})$. See Additional file 1: Table S1 for compounds identification and names. Fig S4. Representative MRM chromatograms of sixteen circulating compounds in plasma. (A) blank plasma, (B) blank plasma spiked with sixteen analytes at LLOQs, (C) rat plasma samples at $0.5 \mathrm{~h}$ after an intravenous 30 min-infusion of Kang-Ai injection. Fig. S5 Effects of several circulating compounds towards eight expressed CYP isozymes and three recombinant UGT enzymes. (A) matrine, (B) ginsenoside Rf, (C) ginsenoside Re, (D) ginsenoside $\mathrm{Rd}$, (E) ginsenoside $\mathrm{Rb} 1$; The probe substrates were incubated at $37^{\circ} \mathrm{C}$ in the absence (control, $0 \mu \mathrm{M}$ ) and presence of tested compounds $(1,10$, and $100 \mu \mathrm{M}$ for matrine; $0.1,1,10$ and $100 \mu \mathrm{M}$ for astragalosides and ginsenosides). Data represent the mean \pm standard deviation of triplicate, ( ${ }^{*}$ compared with those of control, ${ }^{*} p<0.05$ ).
} 


\begin{abstract}
Abbreviations
4-MU: 4-methylumbelliferone; AIC: Akaike information criterion; BCRP: Breast cancer resistance protein; CYP: Cytochrome P450; DDI: Drug-drug interactions; hOCT: Human organic cation transporter; HLM: Human liver microsomes; IS: Internal standard; LLOQ: Lower limit of quantification; MDR1/P-gp: Multidrug resistance protein 1; MRPs: Multidrug resistance-associated proteins; MRM: Multiple reactions monitoring; NADPH: Nicotinamide adenine dinucleotide phosphate; OATP: Organic anion transporting polypeptide; PPD: Protopanaxadiol; PPT: Protopanaxatriol; PK: Pharmacokinetics (PK); QC: Quality control; SC: Schwartz information criterion; UGT: UDP-glucuronosyltransferase; UDPGA: Uridine 5'-diphosphoglucuronosyltransferase; UHPLC-TQD-MS: Ultra high-performance liquid chromatography coupled with a triple quadrupole electrospray tandem mass spectrometry.
\end{abstract}

\section{Acknowledgements}

The authors would like to thank Miss Panpan Yang for the UHPLC-TQD-MS tests.

\section{Authors' contributions}

YJ and YZ conceived and designed the study; QZF performed the pharmacokinetics and enzyme inhibition assays; JMM and XH analyzed the data; QZF, $Y J$ and $Y Z$ wrote this manuscript; $Y Z H, Z X J$ and YXS revised the manuscript. All authors read and approved the final manuscript

\section{Funding}

This work was supported by the National Natural Science Foundation of China (81903704, 81803638), Foundation of He'nan Educational Committee (20A350012), and National Major Scientific and Program of Introducing Talents of Discipline to Universities (B13038).

\section{Availability of data and materials}

Most of the data in the present study are included in this article and its additional information.

\section{Ethics approval and consent to participate}

The animal studies were in accordance with the Guide for the Care and Use of Laboratory Animals (National Institutes of Health). The study protocol was approved by the Ethics Review Committee for Animal Experimentation of Jinan University (Ethical Review NO. 20190301010).

\section{Consent to publish}

Not applicable.

\section{Competing interests}

The authors declare that they have no competing interests

\section{Author details}

${ }^{1}$ Department of Pharmacy, The First Affiliated Hospital of Zhengzhou University, Zhengzhou 450052, China. ${ }^{2}$ Henan Key Laboratory of Precision Clinical Pharmacy, Zhengzhou University, Zhengzhou 450052, China. ${ }^{3}$ Guangdong Province Key Laboratory of Pharmacodynamic Constituents of TCM and New Drugs Research, Jinan University, Guangzhou 510632, China. ${ }^{4}$ College of Pharmacy, Jinan University, Guangzhou 510632, China.

Received: 30 April 2020 Accepted: 26 June 2020

Published online: 06 July 2020

\section{References}

1. Ning ZW, Zhai LX, Peng J, Zhao L, Huang T, Lin CY, Chen WH, Luo Z, Xiao HT, Bian ZX. Simultaneous UPLC-TQ-MS/MS determination of six active components in rat plasma: application in the pharmacokinetic study of Cyclocarya paliurus leaves. Chin Med. 2019;14:28.

2. Yao ZH, Qin ZF, He LL, Wang XL, Dai Y, Qin L, Gonzalez FJ, Ye WC, Yao XS. Identification, bioactivity evaluation and pharmacokinetics of multiple components in rat serum after oral administration of Xian-Ling-Gu-Bao capsule by ultra performance liquid chromatography coupled with quadrupole time-of-flight tandem mass spectrometry. J Chromatogr B. 2017;1041-1042:104-12.
3. Yan R, Yang Y, Chen Y. Pharmacokinetics of Chinese medicines: strategies and perspectives. Chin Med. 2018;13:24.

4. Lu T, Yang J, Gao X, Chen P, Du F, Sun Y, Wang F, Xu F, Shang H, Huang Y, Wang Y, Wan R, Liu C, Zhang B, Li C. Plasma and urinary tanshinol from Salvia miltiorrhiza (Danshen) can be used as pharmacokinetic markers for cardiotonic pills, a cardiovascular herbal medicine. Drug Metab Dispos. 2008;36(8):1578-86.

5. Cheng C, Du F, Yu K, Xu F, Wang F, Li L, Olaleye OE, Yang J, Chen F, Zhong C, Liu Q, Li J, Wang Z, Li C, Xiao W. Pharmacokinetics and disposition of circulating iridoids and organic acids in rats intravenously receiving ReDuNing injection. Drug Metab Dispos. 2016;44(11):1853-8.

6. Lu Q, Li CL. Therapeutic efficacy and safety of Kang-ai injection combined with platinum-based doublet chemotherapy in advanced NSCLC: a meta-analysis. Life Sci. 2018;210:9-19.

7. He ZF, Wang JJ, Wang WD. Effect of Kangý ai injection on serum level of soluble interleukin-2 receptor and vascular endothelial growth factor in patients with esophageal carcinoma during radiotherapy. Chin J Integr Med. 2006;12(4):273-6.

8. Wan YM, Li YH, Xu ZY, Wu HM, Xu Y, Yang M, Wu XN. The effect of transarterial chemoembolization in combination with kang'ai injection on patients with intermediate stage hepatocellular carcinoma: a prospective study. Integr Cancer Ther. 2018;17(2):477-85.

9. Jia M, Zhang B, Qi Y, Yang J, Yao Z, Qin Z, Zhang X, Yao X. Ultra highperformance liquid chromatography coupled with mass spectrometry and chemometric analysis of Kang-Ai injection based on the chemical characterization, simultaneous quantification and relative quantification of forty-seven herbal alkaloids and saponins. J Sep Sci. 2020. https://doi. org/10.1002/jssc.201900878.

10. Yang J, Dai G, Hou Y, Song Z, Wang F, Ji G, Chen J. Quantification of oxymatrine in rat plasma by UPLC-MS/MS to support the pharmacokinetic analyses of oxymatrine-loaded polymersomes. Anal Methods. 2014;6(6):1811-7.

11. Wu XL, Hang TJ, Shen JP, Zhang YD. Determination and pharmacokinetic study of oxymatrine and its metabolite matrine in human plasma by liquid chromatography tandem mass spectrometry. J Pharm Biomed Anal. 2006;41(3):918-24.

12. Xu M, Yin J, Xie L, Zhang J, Zou C, Zou J, Liu F, Ju W, Li P. Pharmacokinetics and tolerance of toal astragalosides after intravenous infusion of astragalosides injection in healthy Chinese volunteers. Phytomedicine. 2013;20(12):1105-11.

13. Jin Y, Guo X, Yuan B, Yu W, Suo H, Li Z, Xu H. Disposition of astragaloside IV via enterohepatic circulation is affected by the activity of the intestinal microbiome. J Agric Food Chem. 2015;63(26):6084-93.

14. Zhang WD, Zhang C, Liu RH, Li HL, Zhang JT, Mao C, Moran S, Chen CL. Preclinical pharmacokinetics and tissue distribution of a natural cardioprotective agent astragaloside IV in rats and dogs. Life Sci. 2006;79(8):808-15.

15. Ran R, Zhang C, Li R, Chen B, Zhang W, Zhao Z, Fu Z, Du Z, Du X, Yang X, Fang Z. Evaluation and Comparison of the Inhibition Effect of astragaloside IV and aglycone cycloastragenol on various UDP-glucuronosyltransferase (UGT) isoforms. Molecules. 2016;21(12):1616.

16. Olaleye OE, Niu W, Du FF, Wang FQ, Xu F, Pintusophon S, Lu JL, Yang JL, Li C. Multiple circulating saponins from intravenous ShenMai inhibit OATP1Bs in vitro: potential joint precipitants of drug interactions. Acta Pharmacol Sin. 2018;40(6):833-49.

17. Yao Z, Li S, Qin Z, Hong X, Dai Y, Wu B, Ye W, Gonzalez FJ, Yao X. Characterization of human UDP-glucuronosyltransferases responsible for glucuronidation and inhibition of norbakuchinic acid, a primary metabolite of hepatotoxicity and nephrotoxicity component bakuchiol in Psoralea corylifolia L. RSC Adv. 2017;7:52661-71.

18. Gao L, Qin Z, Zhang B, Yin Z, Yang XZJ. An investigation of the metabolic activity, isozyme contribution, species differences and potential drugdrug interactions of $\mathrm{PI}-103$, and the identification of efflux transporters for PI-103-Oglucuronide in HeLa1A9 cells. RSC Adv. 2020;10:9610-22.

19. Sun ZH, Chen J, Song YQ, Dou TY, Zou LW, Hao DC, Liu HB, Ge GB, Yang L. Inhibition of human carboxylesterases by ginsenosides: structure-activity relationships and inhibitory mechanism. Chin Med. 2019;14:56.

20. Ge G. Deciphering the metabolic fates of herbal constituents and the interactions of herbs with human metabolic system. Chin J Nat Med. 2019;17(11):801-2. 
21. Lv X, Xia Y, Finel M, Wu J, Ge G, Yang L. Recent progress and challenges in screening and characterization of UGT1A1 inhibitors. Acta Pharm Sin B. 2019;9(2):258-78.

22. Zhou QH, Zhu YD, Zhang F, Song YQ, Jia SN, Zhu L, Fang SQ, Ge GB. Interactions of drug-metabolizing enzymes with the Chinese herb Psoraleae Fructus. Chin J Nat Med. 2019;17(11):858-70.

23. US Food and Drug Administration. Guidance for Industry, Bioanalytical Method Validation; 2013. http://www.fda.gov/downloads/drugs/.

24. Matuszewski BK, Constanzer ML, Chavez-Eng CM. Matrix effect in quantitative LC/MS/MS analyses of biological fluids: a method for determination of finasteride in human plasma at picogram per milliliter concentrations. Anal Chem. 1998;70(5):882-9.

25. Reagan-Shaw S, Nihal M, Ahmad N. Dose translation from animal to human studies revisited. FASEB J. 2008;22(3):659-61.

26. Guo B, Li C, Wang G, Chen L. Rapid and direct measurement of free concentrations of highly protein-bound fluoxetine and its metabolite norfluoxetine in plasma. Rapid Commun Mass Spectrom. 2006;20(1):39-47.

27. He W, Wu JJ, Ning J, Hou J, Xin H, He YQ, Ge GB, Xu W. Inhibition of human cytochrome P450 enzymes by licochalcone A, a naturally occurring constituent of licorice. Toxicol In Vitro. 2015:29(7):1569-76.

28. Xin H, Qi XY, Wu JJ, Wang XX, Li Y, Hong JY, He W, Xu W, Ge GB, Yang L. Assessment of the inhibition potential of Licochalcone A against human UDP-glucuronosyltransferases. Food Chem Toxicol. 2016;90:112-22.

29. Huang LH, Zhong YM, Xiong XH, Cen MF, Cheng XG, Wang GX, Chen JS, Wang $\mathrm{SJ}$. The disposition of oxymatrine in the vascularly perfused rat intestine-liver preparation and its metabolism in rat liver microsomes. J Pharm Sci. 2016;105(2):897-903.

30. Lin P, Dai Y, Yao Z, Qin Z, He L, Wang Q, Wei Z, Zhang Y, Yao X. Metabolic profiles and pharmacokinetics of Qingre Xiaoyanning capsule, a traditional Chinese medicine prescription of Sarcandrae Herba, in rats by UHPLC coupled with quadrupole time-of-flight tandem mass spectrometry. J Sep Sci. 2019;42(4):784-96.

31. Liu XW, Yang JL, Niu W, Jia WW, Olaleye OE, Wen Q, Duan XN, Huang YH, Wang FQ, Du FF, Zhong CC, Li YF, Xu F, Gao Q, Li L, Li C. Human pharmacokinetics of ginkgo terpene lactones and impact of carboxylation in blood on their platelet-activating factor antagonistic activity. Acta Pharmacol Sin. 2018;39(12):1935-46.

32. Davies B, Morris T. Physiological parameters in laboratory animals and humans. Pharm Res. 1993;10(7):1093-5.

33. Halim CE, Xinjing SL, Fan L, Bailey Vitarbo J, Arfuso F, Tan CH, Narula AS, Kumar AP, Sethi G, Ahn KS. Anti-cancer effects of oxymatrine are mediated through multiple molecular mechanism(s) in tumor models. Pharmacol Res. 2019;147:104327.

34. Rashid HU, Xu Y, Muhammad Y, Wang L, Jiang J. Research advances on anticancer activities of matrine and its derivatives: an updated overview. Eur J Med Chem. 2019:161:205-38.

35. Li L, Hou X, Xu R, Liu C, Tu M. Research review on the pharmacological effects of astragaloside IV. Fundam Clin Pharmacol. 2017;31(1):17-36.

36. Mancuso C, Santangelo R. Panax ginseng and Panax quinquefolius: from pharmacology to toxicology. Food Chem Toxicol. 2017;107(Pt A):362-72.

37. Liu W, Shi J, Zhu L, Dong L, Luo F, Zhao M, Wang Y, Hu M, Lu L, Liu Z. Reductive metabolism of oxymatrine is catalyzed by microsomal CYP3A4. Drug Des Devel Ther. 2015;9:5771-83.
38. Yang Z, Gao S, Yin T, Kulkarni KH, Teng Y, You M, Hu M. Biopharmaceutical and pharmacokinetic characterization of matrine as determined by a sensitive and robust UPLC-MS/MS method. J Pharm Biomed Anal. 2010;51(5):1120-7.

39. Evans WE, Relling MV. Pharmacogenomics: translating functional genomics into rational therapeutics. Science. 1999;286(5439):487.

40. Miners JO, Mackenzie PI, Knights KM. The prediction of drug-glucuronidation parameters in humans: uDP-glucuronosyltransferase enzymeselective substrate and inhibitor probes for reaction phenotyping and in vitro-in vivo extrapolation of drug clearance and drug-drug interaction potential. Drug Metab Rev. 2010;42(1):196-208.

41. Seong SJ, Kang WY, Heo JK, Jo J, Choi WG, Liu KH, Lee S, Choi MK, Han YH, Lee HS, Ohk B, Lee HW, Song IS, Yoon YR. A comprehensive in vivo and in vitro assessment of the drug interaction potential of red ginseng. Clin Ther. 2018;40(8):1322-37.

42. Pan $X$, Wang L, Gründemann $D$, Sweet $D H$. Inhibition of human organic cation transporters by the alkaloids matrine and oxymatrine. Fitoterapia. 2014;92:206-10.

43. Li X, Hu J, Wang B, Sheng L, Liu Z, Yang S, Li Y. Inhibitory effects of herbal constituents on P-glycoprotein in vitro and in vivo: herb-drug interactions mediated via P-gp. Toxicol Appl Pharmacol. 2014;275(2):163-75.

44. Zhang G, Ou R, Li F, Wu J, Zheng L, Tong Y, Liu Y, Liu Z, Lu L. Regulation of drug-metabolizing enzymes and efflux transporters by Astragali radix decoction and its main bioactive compounds: implication for clinical drug-drug interactions. J Ethnopharmacol. 2016;180:104-13.

45. Zhang W, Liu M, Yang L, Huang F, Lan Y, Li H, Wu H, Zhang B, Shi H, Wu X. P-glycoprotein Inhibitor tariquidar potentiates efficacy of astragaloside IV in experimental autoimmune encephalomyelitis mice. Molecules. 2019:24(3):561.

46. Zhan T, Yao N, Wu L, Lu Y, Liu M, Liu F, Xiong Y, Xia C. The major effective components in Shengmai Formula interact with sodium taurocholate co-transporting polypeptide. Phytomedicine. 2019;59:152916.

47. Lee S, Kwon M, Choi MK, Song IS. Effects of red ginseng extract on the pharmacokinetics and elimination of methotrexate via Mrp2 regulation. Molecules. 2018;23:2948.

48. Li N, Wang D, Ge G, Wang X, Liu Y, Yang L. Ginsenoside metabolites inhibit P-glycoprotein in vitro and in situ using three absorption models. Planta Med. 2014:80(4):290-6.

49. Zhang Q, Li FJ, Jin RM, Song ZP. Study on the hepatotoxicity induced by matrine and oxymatrine. Chin Arch Tradit Chinese Medicine. 2011;29:1222-5.

\section{Publisher's Note}

Springer Nature remains neutral with regard to jurisdictional claims in published maps and institutional affiliations.

\footnotetext{
Ready to submit your research? Choose BMC and benefit from:

- fast, convenient online submission

- thorough peer review by experienced researchers in your field

- rapid publication on acceptance

- support for research data, including large and complex data types

- gold Open Access which fosters wider collaboration and increased citations

- maximum visibility for your research: over $100 \mathrm{M}$ website views per year
}

At BMC, research is always in progress.

Learn more biomedcentral.com/submissions 